\title{
Phosphatidylinositol 3-kinase signaling determines kidney size
}

\author{
Jian-Kang Chen, ${ }^{1}$ Kojiro Nagai, ${ }^{2}$ Jianchun Chen, ${ }^{2}$ David Plieth, ${ }^{2}$ Masayo Hino, ${ }^{2}$ Jinxian Xu, ${ }^{1}$ Feng Sha, ${ }^{2}$ T. Alp Ikizler, ${ }^{2}$ \\ C. Chad Quarles, ${ }^{3}$ David W. Threadgill, ${ }^{4}$ Eric G. Neilson, ${ }^{5}$ and Raymond C. Harris ${ }^{2}$ \\ 'Departments of Cellular Biology and Anatomy and Medicine, Medical College of Georgia, Georgia Regents University, Augusta, Georgia, USA. ${ }^{2}$ Department of Medicine and ${ }^{3}$ Vanderbilt University \\ Institute of Imaging Science and Department of Radiology and Radiological Sciences, Vanderbilt University School of Medicine, Nashville, Tennessee, USA. ${ }^{4}$ Department of Veterinary Pathobiology, \\ College of Veterinary Medicine and Biomedical Sciences, and Department of Molecular and Cellular Medicine, College of Medicine, Texas A\&M University, College Station, Texas, USA. \\ ${ }^{5}$ Department of Medicine and Cell and Molecular Biology, Feinberg School of Medicine, Northwestern University, Chicago, Illinois, USA.
}

\begin{abstract}
Kidney size adaptively increases as mammals grow and in response to the loss of 1 kidney. It is not clear how kidneys size themselves or if the processes that adapt kidney mass to lean body mass also mediate renal hypertrophy following unilateral nephrectomy (UNX). Here, we demonstrated that mice harboring a proximal tubule-specific deletion of Pten $\left(P\right.$ ten $\left.^{\text {ptKo }}\right)$ have greatly enlarged kidneys as the result of persistent activation of the class I PI3K/mTORC2/AKT pathway and an increase of the antiproliferative signals p21 $1^{\mathrm{Cip} 1 / \mathrm{WAF}}$ and p27 $7^{\mathrm{Kip} 1}$. Administration of rapamycin to Pten ${ }^{\text {tho }}$ mice diminished hypertrophy. Proximal tubule-specific deletion of Egfr in Pten ${ }^{p t K o}$ mice also attenuated class I PI3K/mTORC2/AKT signaling and reduced the size of enlarged kidneys. In Pten ${ }^{\text {ptKo }}$ mice, UNX further increased mTORC1 activation and hypertrophy in the remaining kidney; however, mTORC2-dependent AKT phosphorylation did not increase further in the remaining kidney of $P_{t e n}^{\text {ptKo }}$ mice, nor was it induced in the remaining kidney of WT mice. After UNX, renal blood flow and amino acid delivery to the remaining kidney rose abruptly, followed by increased amino acid content and activation of a class III PI3K/mTORC1/S6K1 pathway. Thus, our findings demonstrate context-dependent roles for EGFR-modulated class I PI3K/mTORC2/AKT signaling in the normal adaptation of kidney size and PTEN-independent, nutrient-dependent class III PI3K/mTORC1/S6K1 signaling in the compensatory enlargement of the remaining kidney following UNX.
\end{abstract}

\section{Introduction}

Kidney mass in growing mammals adapts concordantly to lean body mass (1). Much of this kidney mass comprises proximal tubules. Unilateral nephrectomy (UNX) in rodents and humans also results in compensatory increases in the size of the remaining kidney (2), and post-nephrectomy transplantation of a hypertrophied kidney restores both the transplanted kidney and the native, hypertrophied contralateral kidney to their normal size (3). This tight autoregulation of native kidney mass to the metabolic demands of body mass has long intrigued cell biologists, even though an understanding of the underlying adaptive mechanisms has eluded decades of study (2-4).

Phosphatase and tensin homolog (PTEN) is a tumor-suppressor gene that is frequently mutated or deleted somatically in a variety of human cancers (5). The PTEN gene encodes a cytoplasmic protein that shares significant homology to the catalytic domain of protein phosphatases and to the cytoskeletal proteins tensin and auxilin (5). PTEN has both protein phosphatase and lipid phosphatase activity (6). The protein phosphatase activity of PTEN inhibits cell migration, spreading, and focal adhesions (7). While the lipid phosphatase activity of PTEN is critical for its

\section{Related Commentary: p. 2267}

Conflict of interest: The authors have declared that no conflict of interest exists. Submitted: September 16, 2014; Accepted: March 17, 2015.

Reference information: J Clin Invest. 2015;125(6):2429-2444. doi:10.1172/JCI78945. tumor-suppressor function, the activity of PTEN toward protein substrates is not essential for growth suppression $(8,9)$.

A primary physiological lipid substrate of PTEN is phosphotidylinositol-3,4,5-triphosphate (PIP3) (9), a lipid second-messenger molecule generated upon activation of the class I phosphatidylinositol 3-kinases (PI3K) in response to various stimuli, including growth factors and cytokines. Thus, PTEN negatively regulates class I PI3K signaling by dephosphorylating the D3-position phosphate of the inositol ring of PIP3 (8-10). PTEN deficiency results in accumulation of PIP3, which recruits the serine/threonine kinase $\mathrm{AKT}$, also called protein kinase $\mathrm{B}(\mathrm{PKB})$, to the plasma membrane where AKT is phosphorylated and fully activated by upstream kinases, including the phosphoinositide-dependent kinase 1 (PDK1) $(11,12)$ and the mechanistic (formerly mammalian) target of rapamycin (mTOR) complex 2 (mTORC2) (13). PTEN specifically inhibits class I PI3K activity and does not interfere with activation or signaling of class II or III PI3K. Although class II PI3K is not very well studied, some investigators report an adaptor role in clathrin-mediated endocytosis (14). Class III PI3K in eukaryotes is conserved from yeast to humans. Studies suggest that class III $\mathrm{PI} 3 \mathrm{~K}$, in association with other proteins, regulates membrane trafficking, endosomal protein sorting, autophagy, and cytokinesis (14). There are early findings that class III PI3K also modulates amino acid sensing, leading to mTORC1 activation (15).

mTOR forms 2 structurally and functionally distinct multiprotein complexes in all mammalian cells: mTOR complex 1 
A

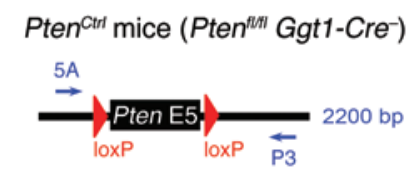

Pten ${ }^{p / K O}$ mice (Pten ${ }^{n ! \prime \prime}$ Ggt1-Cre $)$

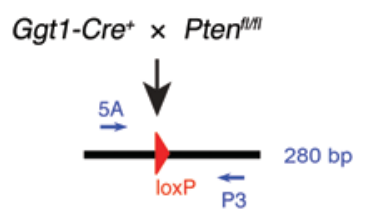

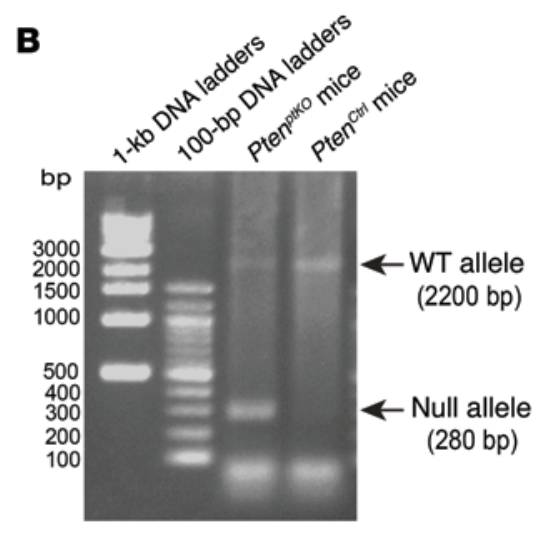

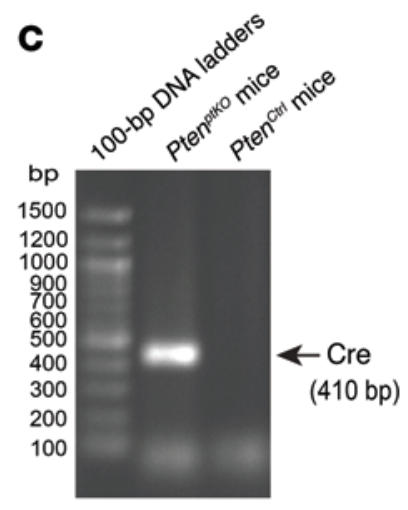

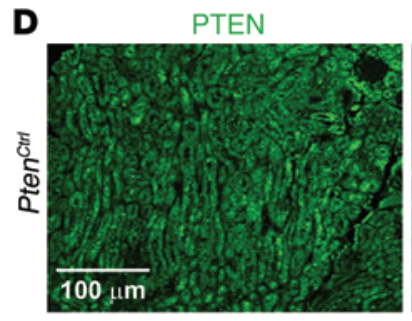
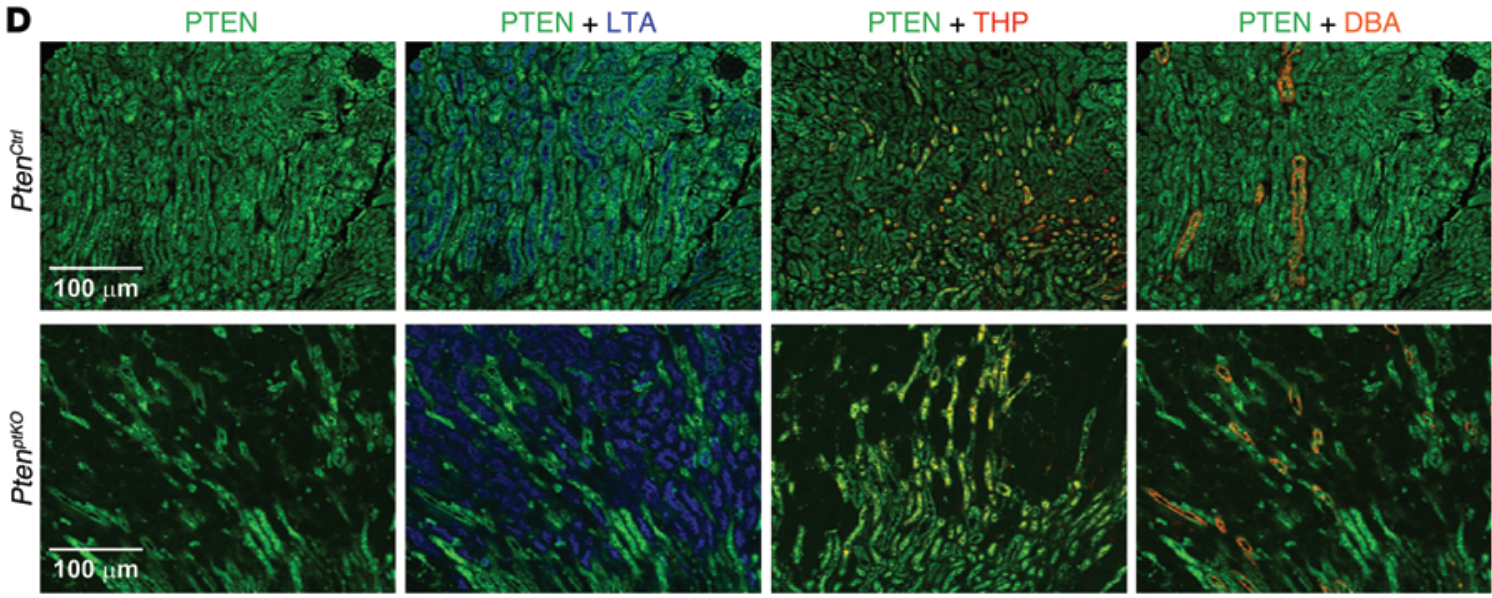

Figure 1. Characterization of Pten ${ }^{\text {ptKo }}$ mice. (A-C) Generation of renal proximal tubular cell-specific Pten-KO (Pten ${ }^{p t K o}$ ) mice. (A) Schematic depicting the generation of $P$ ten $^{p t K o}$ mice. Gender-matched $P$ ten $^{f / f l}$ littermates were used as controls (Pten ${ }^{(t r)}$ ). (B) PCR verification using the primer pairs $5 A$ and P3 (their relative positions are indicated in $\mathbf{A}$ and their sequences listed in Methods), with whole-kidney genomic DNA as templates. The 2200-bp band from the WT allele was readily apparent in Pten ${ }^{c t r l}$ mice but only faintly detected in $P$ ten $^{\text {ptKo }}$ mice. The 280-bp band was detected only in $P$ ten ${ }^{\text {ptKo }}$ mice but not in control mice. (C) The Cre recombinase gene was detected by PCR as a 410-bp band in Pten ${ }^{\text {ptKo }}$ mice but not in Pten ${ }^{\text {ctrl }}$ mice, with genomic DNA from ear-punch biopsy samples used as templates. (D) Immunofluorescence staining of kidney sections confirmed that the Ggt1-Cre-mediated Pten deletion occurred selectively in the LTA-positive area (blue) but not in the THP- or DBA-positive tubules (red) of Pten ${ }^{\text {ptKo }}$ mice, while PTEN (green) was ubiquitously expressed in the kidneys of Pten ${ }^{\text {ctrl }}$ mice. Scale bars: $100 \mu \mathrm{m}$.

(mTORC1) and mTORC2 (16). The regulatory-associated protein of mTOR (RAPTOR) only exists in mTORC1, while the rapamycin-insensitive companion of mTOR (RICTOR) is the key component of mTORC2. Increased mTORC1 activity not only stimulates ribosome biogenesis and protein synthesis but also modulates a range of cellular activities that are essential for cell growth (16). Unlike mTORC1, mTORC2 does not regulate the phosphorylation of S6 kinase 1 (S6K1) and eukaryotic translation initiation factor (eIF) 4E-binding protein 1 (4E-BP1) (17); instead, mTORC2 regulates the class I PI3K signaling pathway by directly phosphorylating AKT on the key residue Ser473, and this phosphorylation is required for full activation of AKT (13). AKT activation inhibits apoptosis and stimulates cell proliferation in many cell types by phosphorylating multiple downstream targets (18).

Of note, tuberous sclerosis complex (TSC) is an autosomaldominant syndrome characterized by the development of hamartomas in a wide range of organs, including the kidneys, and is caused by mutation of either the TSC1 or TSC2 tumor-suppressor gene (19). The TSC1 and TSC2 proteins, along with Tre2-
Bub2-Cdc16-1 domain family member 7 (TBC1D7), form a functional complex in which TSC2 is the catalytic subunit possessing GTPase-activating protein (GAP) activity toward RHEB, a member of the RAS family GTPases (20). Genetic and biochemical evidence indicates that RHEB functions downstream of TSC2 and upstream of mTORC1 (16). Activation of AKT phosphorylates and inactivates the GAP activity of TSC2 toward RHEB, resulting in accumulation of the GTP-bound form of RHEB and the consequent activation of the mTORC1 pathway $(16,21)$. We have previously demonstrated that activation of the rapamycin-sensitive mTORC1/S6K1 signaling pathway in the remaining kidney in response to contralateral nephrectomy mediates increased RNA and protein synthesis in compensatory renal hypertrophy $(22,23)$. A recent study demonstrated that phosphorylated ribosomal protein S6 (p-rpS6) is a downstream effector of the mTORC1/S6K1 signaling pathway mediating renal hypertrophy (24).

Increasing evidence highlights the importance of PTEN-regulated signaling in numerous biological processes. Recent studies suggest a role in shaping the pattern of epithelial branching morpho- 
A
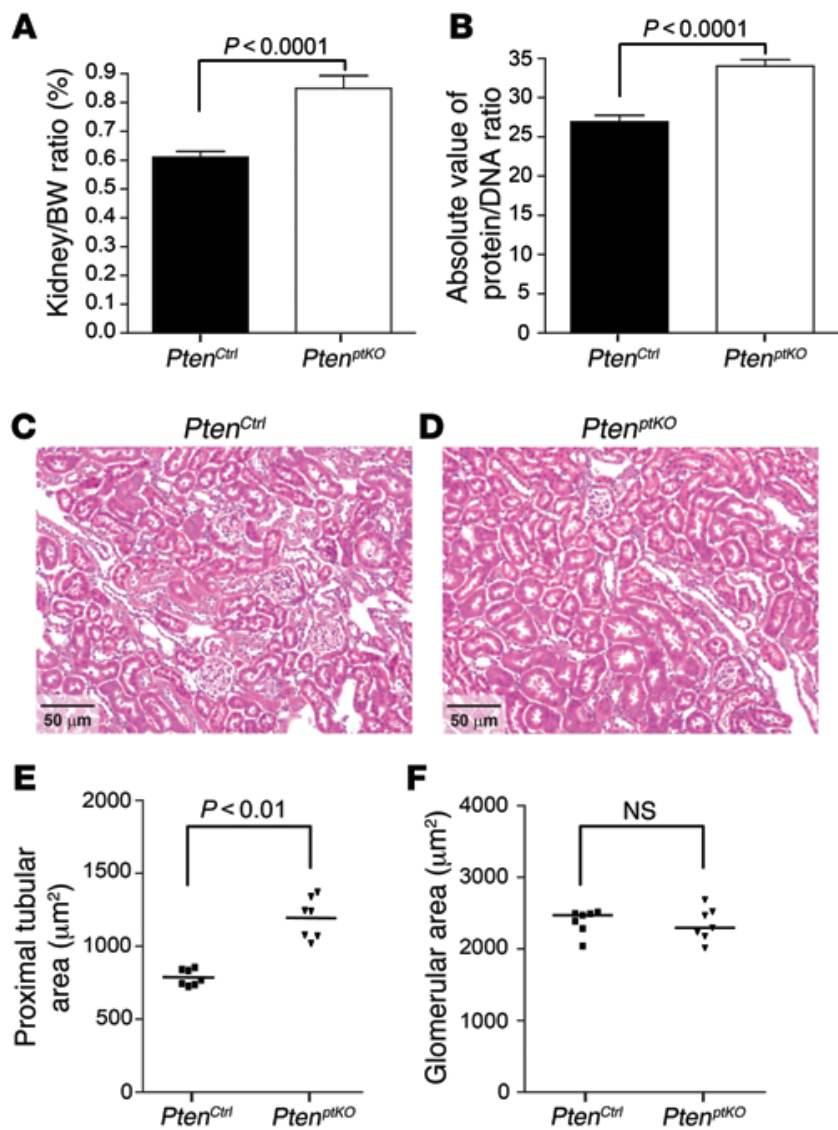

genesis in the developing kidneys (25). However, the physiological role of PTEN in the nephron segments of developed, mature kidneys remains unclear. Homozygous deletion of Pten is embryonically lethal before midgestation (26). Therefore, we used a Cre/LoxP strategy to delete Pten specifically in renal proximal tubules (Ggt1Cre Pten ${ }^{f / f l}$, herein referred to as Pten $\left.{ }^{p t K O}\right)(27,28)$, making use of the promoter Ggt1, which is not active in that nephron segment until nephrogenesis is virtually complete $(28,29)$.

Here, we found that Pten ${ }^{p t K o}$ mice developed significantly larger kidneys than did control littermates, an enlargement characterized by a predominance of proximal tubule cell hypertrophy, with only slightly increased cell proliferation. These results indicate an important role for PTEN in the class I PI3K/mTORC2/ AKT/mTORC1/S6K1 pathway in regulating adaptive kidney size; genetic deletion of Egfr in Pten ${ }^{p t K O}$ mice also disrupted this signaling pathway. Unexpectedly, we found that deletion of Pten did not affect compensatory hypertrophy after contralateral nephrectomy. Instead, compensatory hypertrophy was associated with increased activation of PTEN-independent, nutrient-dependent class III PI3K/mTORC1/S6K1 signaling.

\section{Results}

Selective Pten deletion in renal proximal tubules. Significant expression of $\gamma \mathrm{GT}$ (encoded by Ggt1) occurs in the renal proximal tubule beginning 2-3 weeks after birth, when nephrogenesis is virtually complete (29). The Ggt1 promoter has been used to successfully target different genes of interest specifically to the renal proximal tubule (28). We have previously generated a transgenic mouse
Figure 2. Pten deletion in renal proximal tubules induces renal hypertrophy. Renal proximal tubular cell-specific Pten deletion increased the kidney/BW (A) and protein/DNA (B) ratios in the kidney. Renal histology

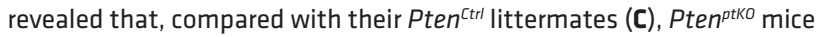
(D) showed hypertrophy in renal proximal tubules but not in glomeruli. Morphometric analysis of the cross-sectional tubule areas and glomerular areas confirmed hypertrophic renal growth exclusively in the proximal tubules (E) but not in the glomeruli (F) of $P$ ten $^{p t K o}$ mice. Images in $\mathbf{C}$ and D are representative of $P$ ten $^{\text {Ctrl }}$ and $P$ ten $^{p t K o}$ mice at 8 weeks and 4 days of age. A 1-tailed, unpaired $t$ test was used for statistical analysis of the data in $\mathbf{A}$ and $\mathbf{B}$; Mann-Whitney $U$ tests were used for statistical analysis of the data in $\mathbf{E}$ and $\mathbf{F}$. $n=7$ mice per group with similar results. $P$ values are indicated in the respective figures. Scale bars: $50 \mu \mathrm{m}$.

line expressing Cre recombinase under the control of the Ggt1 promoter (28). As indicated in Figure 1A, to generate a renal proximal tubule-specific Pten-KO mouse line (Pten $\left.{ }^{p t K O}\right)$, we crossed this Ggt1-Cre mouse with Pten exon 5-floxed mice (Pten $\left.{ }^{f / f f}\right)$; exon 5 encodes the essential phosphatase core domain of the PTEN protein (27). PCR of genomic DNA from renal cortex (the bulk of which is made up of proximal tubules) indicated the expected 280-bp band amplified from the null allele, with significantly decreased expression of the 2200-bp band from the WT allele of the Pten gene in Pten ${ }^{p t K O}$ mice, although we detected a residual 2200-bp band signal (presumably amplified from the WT allele of the Pten gene in other cell types such as the distal tubular cells in the renal cortex). The 280-bp band signal representing the null allele was not detectable in Pten $^{f / f l}$ mice (herein referred to as Pten $^{\mathrm{Ct}}{ }^{\mathrm{t}}$ ) (Figure 1B). PCR of the genomic DNA from renal cortex detected Cre recombinase only in Pten ${ }^{p t K o}$ mice but not in their WT littermate control (Pten ${ }^{\mathrm{Ctr}}$ ) mice (Figure 1C), confirming that Pten $^{p t K o}$ mice underwent Ggt1-Cre-mediated recombination successfully. Immunofluorescence staining, as indicated by the lack of PTEN colocalization with the proximal tubule-specific lectin Lotus tetragonolobus agglutinin (LTA) in Pten ${ }^{p t K o}$ kidneys, but appropriate colocalization to the thick ascending limb (Tamm-Horsfall protein [THP]) and collecting duct (Dolichos biflorus agglutinin [DBA]), confirmed that the Ggt1-Cre-mediated Pten deletion was largely confined to renal proximal tubules (Figure 1D).

Pten deletion in renal proximal tubules induced renal hypertrophy. Kidneys from Pten ${ }^{p t K O}$ mice were morphologically indistinguishable from those of Pten ${ }^{\mathrm{Ctrl}}$ mice during the first few weeks of postnatal life. However, by 5 to 6 weeks of age, there was progressive kidney enlargement in Pten ${ }^{p t K o}$ homozygous, but not heterozygous, mice. As indicated in Figure 2, by 8 weeks of age, Pten ${ }^{p t K o}$ mice exhibited marked renal growth characterized by increased kidney/ BW (Figure 2A) and protein/DNA (Figure 2B) ratios. Renal histology revealed that compared with their WT littermate $\mathrm{Pten}^{\mathrm{Ctrl}}$ mice (Figure 2C), Pten $^{p t K o}$ mice showed markedly enlarged renal proximal tubules without glomerular enlargement (Figure 2D). Morphometric analyses of cross-sectional tubules and glomerular areas confirmed significant increases in renal mass exclusively in the proximal tubules (Figure 2E), but not in the glomeruli (Figure 2F).

Pten deletion in renal proximal tubules stimulated minor but statistically significant cell proliferation. Although the exact mechanism that activates mTORC2 kinase activity is not completely understood, recent studies reported that PIP3, which accumulates when Pten is deleted, directly stimulates the kinase activity of 

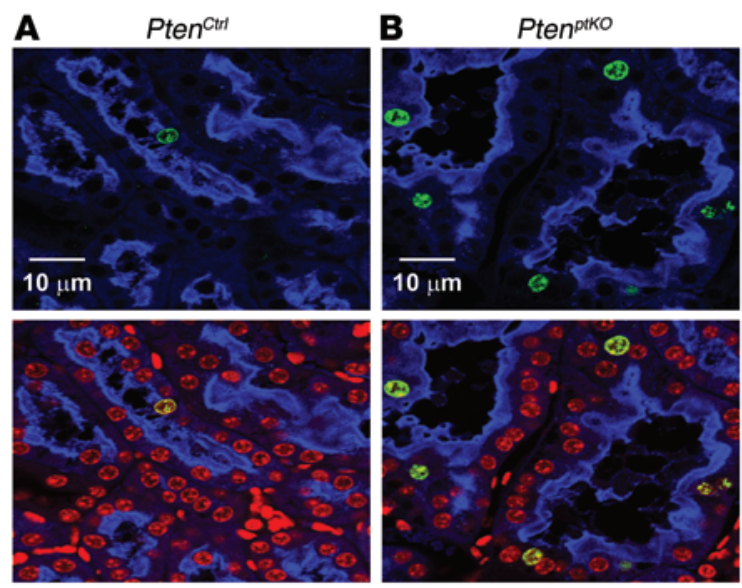

\section{C.}

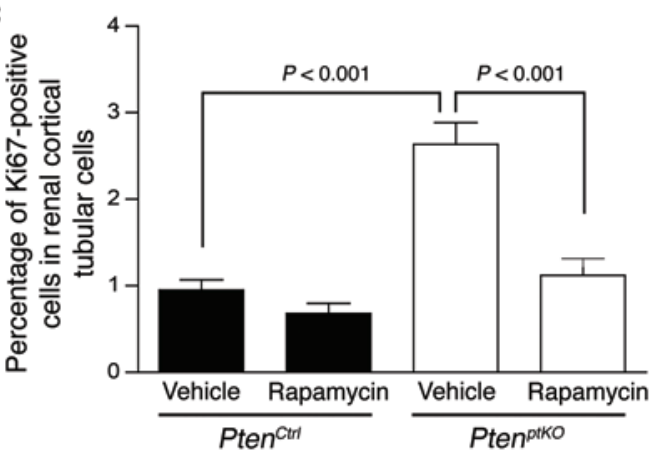

mTORC2 (30). The mTORC2 kinase activity then directly phosphorylates AKT at Ser473, which, along with AKT phosphorylation at Thr308 by PDK1 (11), is required for full activation of AKT (13). In addition to mediating insulin signaling, AKT activation induces cell proliferation in some cases (18). Of note, although increased protein synthesis, and thereby cell growth, is the predominate factor causing renal hypertrophy in response to removal of the contralateral kidney, cell proliferation is also a minor contributing factor in compensatory renal hypertrophy (31).

To this end, we evaluated cell proliferation by immunofluorescence staining for the proliferating cell marker Ki67. As shown in Figure 3, compared with the kidneys of their WT Pten ${ }^{\text {Ctrl }}$ littermates (Figure 3A), Pten $^{p t K o}$ kidneys had a statistically significant increase in Ki67-positive proliferating cells (Figure 3, B and C). Rapamycin treatment of Pten ${ }^{p t K O}$ mice significantly decreased Pten deletion-induced cell proliferation compared with that seen in vehicle-treated Pten ${ }^{p t K O}$ mice (Figure 3C)

Pten deletion in renal proximal tubules did not cause development of tumors in aging kidney. Since enlarged kidneys from Pten ${ }^{\text {ptKO }}$

Figure 4. Renal proximal tubular cell-specific Pten deletion activates the AKT/TSC2/mTORC1 signaling pathway. Pten ${ }^{C t r l}$ and $P$ ten $^{p t K O}$ mice were sacrificed at 9 weeks of age, and their kidney lysates were subjected to immunoblot analysis with the indicated phospho-specific antibodies (A-C, top and middle panels), followed by stripping and reprobing of the same blot in each subset of $\mathbf{A}-\mathbf{C}$ with an antibody against $\beta$-actin (A, bottom) or against the corresponding total proteins (B and $\mathbf{C}$, bottom) to ensure equal loading. Each lane represents 1 sample from the left kidney of an individual mouse. Shown are representative blots for 5 mice per genotype group with similar results.
Figure 3. Pten deletion in renal proximal tubules stimulates slight but statistically significant cell proliferation. (A and B) Representative images of immunofluorescence staining for Ki67 (green), a marker of cell proliferation, in the LTA-positive proximal tubules (blue) in kidney sections from Pten ${ }^{C t r l}$ mice (A) and Pten ${ }^{p t K o}$ mice (B), with DAPI staining of nuclei (red). (C) Compared with vehicle treatment, rapamycin treatment significantly inhibited the increased cell proliferation seen in Pten ptKo mice. Six images were randomly taken of the cortical tubule area in $P \operatorname{ten}^{c t r l}$ or $P$ ten $^{\text {ptKo }}$ mice (original magnification, $\times 200$ ) for the quantification of proliferating cells. Values are presented as the ratio of Ki67-positive tubular nuclei (highlighted in green in B by Ki67 staining) to the total nuclei number (highlighted in red in $\mathbf{A}$ and $\mathbf{B}$ by DAPI staining) in renal proximal tubules (highlighted in blue in $\mathbf{A}$ and $\mathbf{B}$ by LTA staining). ANOVA with Bonferroni's $t$ correction was used for statistical analysis of the data in C. $n=4-6$ mice per group. $P$ values are indicated in the respective figures. Scale bars: $10 \mu \mathrm{m}$.

mice appeared otherwise normal, we followed a cohort of Pten ${ }^{\text {ptKo }}$ mice for over a year. However, none of the Pten ${ }^{\text {ptKo }}$ mice developed any detectable renal tumors, even when another cohort of Pten $^{\text {ptKO }}$ mice was monitored up to 78 weeks of age, although hepatocyte-specific Pten deletion caused $100 \%$ of the mice to develop adenomas or hepatocellular carcinomas by this age (32).

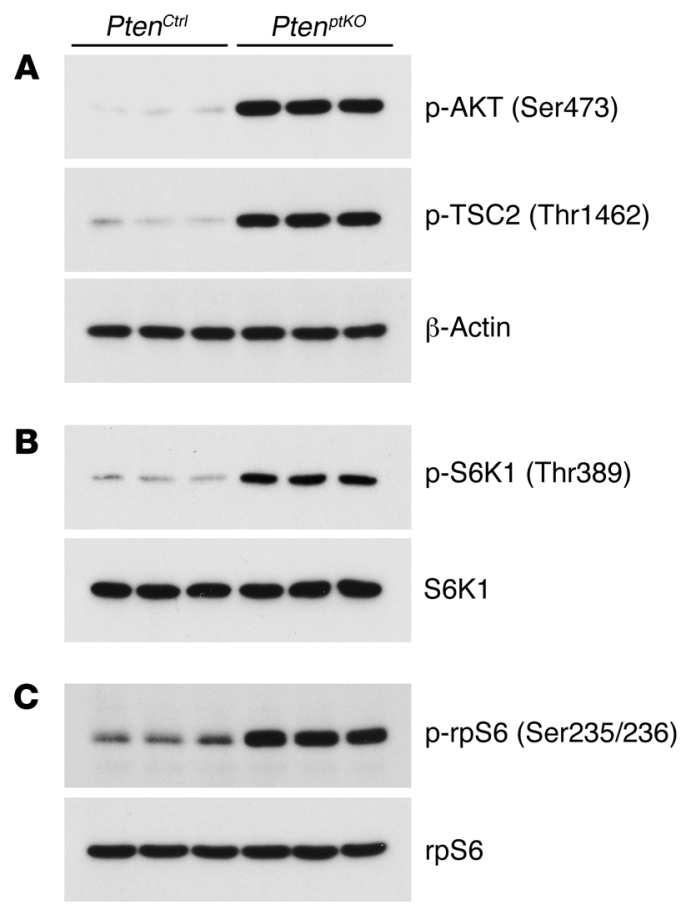


A
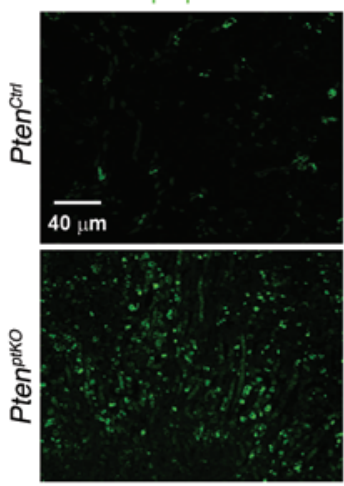

B
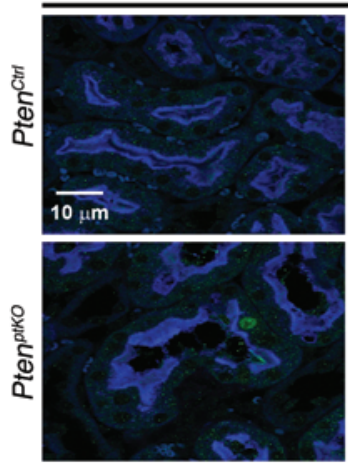

C

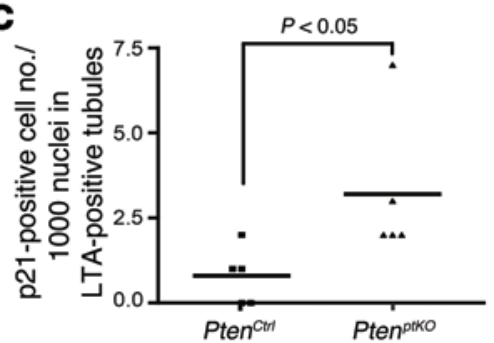

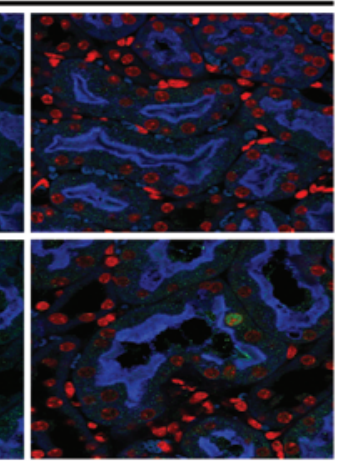

p-rpS6 + LTA
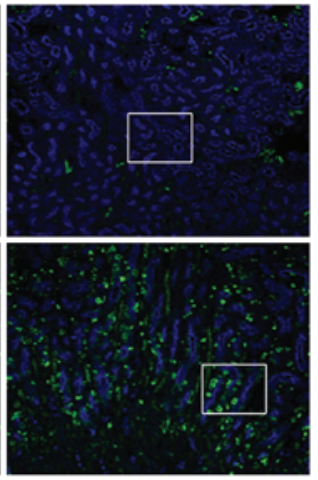

21/LTA/Nuclei

Pten prko
p-rpS6 + LTA

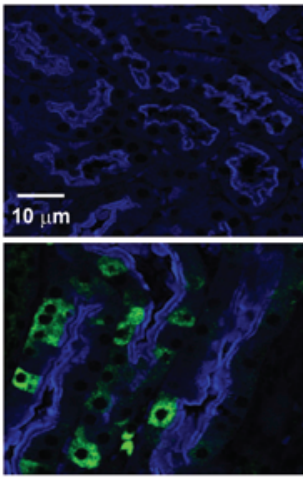

D

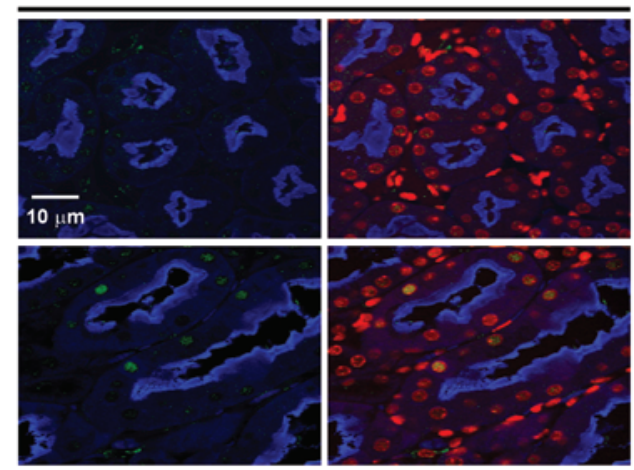

E

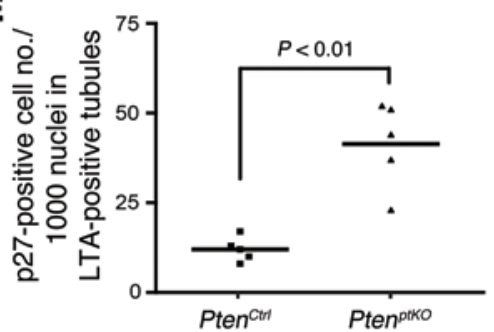

Figure 5. Renal proximal tubule-specific Pten KO increases rpS6 phosphorylation and p21- or p27-positive cells in LTA-positive renal proximal tubules. (A) Localization of increased rpS6 phosphorylation in Pten ${ }^{\text {ptKo }}$ kidneys. Kidney sections were stained with an antibody against S235/236-phosphorylated rpS6 (p-rpS6) and the renal proximal tubule marker LTA. Representative images at $\times 100$ original magnification (left 2 panels) indicating increased p-rpS6 (green) in Pten ${ }^{p t K o}$ kidneys (left 2 lower panels) compared with Pten ${ }^{\text {ctrl }}$ kidneys (left 2 top panels). Images at $\times 400$ original magnification (third panels, top and bottom rows), along with their respective phase-contrast images (fourth panels, top and bottom rows), confirmed the localization of increased p-rpS6 staining in the cytoplasm of renal proximal tubules (with LTA staining on the brush-border membranes). (B-E) Pten ${ }^{\text {ptKo }}$ renal proximal tubule cells exhibited increased protein expression of the cell cycle inhibitors $\mathrm{p} 21^{\mathrm{cip} 1 / \mathrm{WAF}}$ and $\mathrm{p} 27^{\mathrm{Kip} 1}$. Triple immunofluorescence staining revealed significant increases

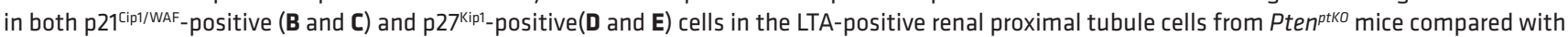
those from Pten ${ }^{c t r l}$ mice, respectively. Shown are representative images from 5 individual mice per genotype group with similar results. For quantitation of p21 $1^{\text {ip/ } / \text { WAF }}$-positive (C) and p27 Kip1-positive (E) cells per 1,000 nuclei in LTA-positive tubules, Mann-Whitney $U$ tests were used for statistical analysis of the data, with the indicated $P$ values from 5 mice per group. Scale bars: $40 \mu \mathrm{m}$ (left 2 panels of $\mathbf{A}$ ) and $10 \mu \mathrm{m}$ (right 2 panels of $\mathbf{A}, \mathbf{B}$, and $\mathbf{D}$ ).

Pten deletion activated both mTORC2/AKT and mTORC1/ S6K1 signaling. PTEN is known to dephosphorylate the lipid second messenger PIP3, which positively regulates AKT activity (10), and overexpression of active AKT in the heart results in massive cardiac hypertrophy (33). We found that Ser473-phosphorylated AKT was markedly increased in the kidneys of Pten ${ }^{p t K O}$ mice (Figure 4A), indicating mTORC2 activation (13). Thr308-phosphorylated AKT levels were also increased (data not shown). TSC2 is a physiological substrate of AKT in vivo, and AKT phosphorylation of Thr1462 inhibits the engagement of RHEB by TSC2, thus acti- vating mTORC1 (16). Thr1462-phosphorylated TSC2 levels were also markedly increased in the kidneys of Pten ${ }^{p t K O}$ mice (Figure 4A), thus further confirming activation of mTORC2. The levels of both rapamycin-sensitive Thr389-phosphorylated S6K1 (Figure 4B) and downstream Ser235/236-phosphorylated rpS6 were also increased in the kidneys of Pten ${ }^{p t K O}$ mice (Figure 4C). Double immunofluorescence staining confirmed that the increased rpS6 phosphorylation was localized to the cytoplasm of renal tubular epithelial cells that costained with LTA, thus confirming MTORC1 activation in the renal proximal tubules of Pten ${ }^{p t K O}$ mice (Figure $5 \mathrm{~A}$ ). 


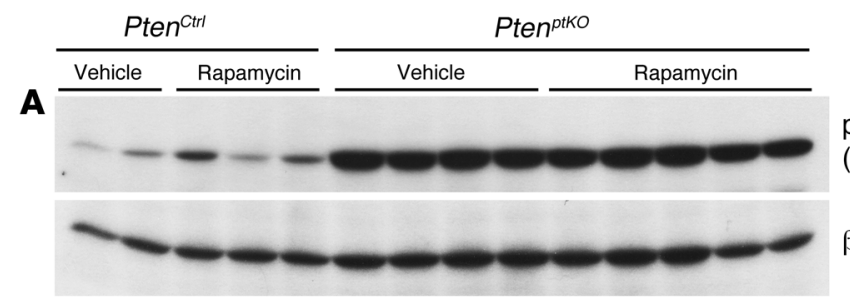

p-AKT

(Ser473)

$\beta$-Actin

B

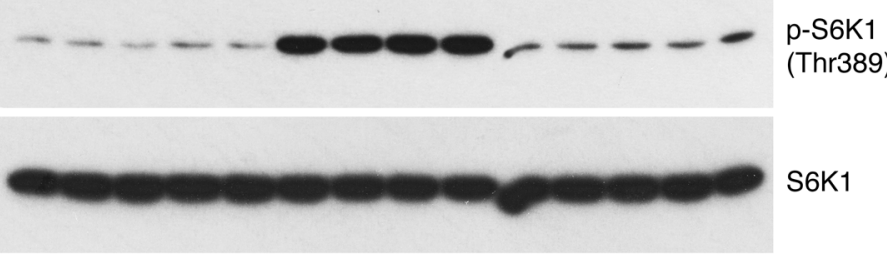

C
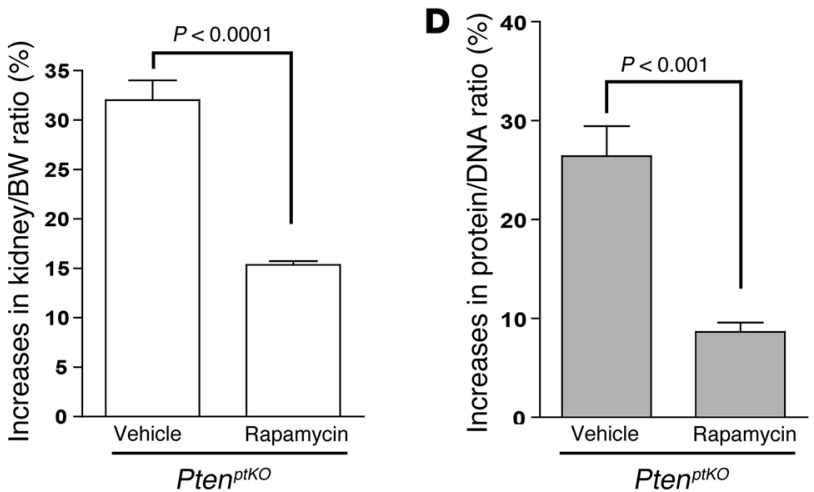

Figure 6. Rapamycin inhibits mTORC1 signaling and renal growth in Pten ${ }^{\text {ptKo }}$ mice. Ten-week-old $P$ ten $^{p t K o}$ mice were treated with vehicle alone or rapamycin via daily i.p. injection (1 mg/kg BW) for 7 days. Rapamycin treatment had no effect on AKT phosphorylation (A) but inhibited S6K1 phosphorylation (B). Rapamycin also significantly attenuated the increases in kidney/BW (C) and protein/DNA (D) ratios in Pten ${ }^{p t K o}$ mice. A 1-tailed, unpaired $t$ test was used for statistical analysis of the data in $\mathbf{C}$ and D. $n=5$ mice per group. $P$ values are indicated in the respective figures.

mediated AKT phosphorylation at Ser473 (Figure 6A) or PDK1-mediated AKT phosphorylation at Thr308 (data not shown), but almost completely blocked mTORC1-mediated S6K1 phosphorylation at Thr389 (Figure 6B), attenuated the increase in the kidney/BW ratio (Figure 6C), and decreased the protein/DNA ratio (Figure 6D).

Egfr deletion attenuated mTORC2/AKT/mTORC1/ S6K1 signaling and reduced Pten KO-induced kidney hypertrophy. Mouse proximal tubular cells express EGFR (38), and activation of this receptor tyrosine kinase by various ligands (EGF, proHB-EGF, TGF $\alpha$, or amphiregulin) is implicated in the activation of class I PI3K/AKT signaling (2). In addition, EGFR activation by proHBEGF is implicated in angiotensin II-induced hypertrophy of renal epithelial cells in culture (39). We crossed Egfr ${ }^{f l / f l}$ mice with Ggt1-Cre mice (28) and produced proximal tubule-specific Egfr-KO (Ggt1-Cre Egfrt/fl, herein referred to as $\left.E g f r^{p t K O}\right)$ mice, which are fertile and have no apparent phenotype (40). To determine whether EGFR is part of the PTEN/class I PI3K/mTORC2/ $\mathrm{AKT} / \mathrm{mTORC} 1$ axis involved in regulating renal size in vivo, we crossed Egfr ${ }^{p t K O}$ mice with $P t e n^{f l / f l}$ mice to produce Egfrfl/fl Pten ${ }^{f l / f l}$ and Egfr ${ }^{f l / f l}$ Pten $^{f l /+}$ Ggt1-Cre mice, which were then intercrossed to generate renal proximal tubule-specific Egfr and Pten double-KO (Ggt1-Cre Egfrfl/fl Pten ${ }^{f l f l}$, herein referred to as Egfr Pten ${ }^{\text {ptDKO }}$ ) mice

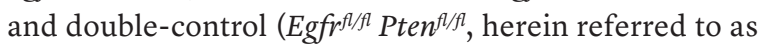
Egfr Pten ${ }^{\text {DCtrl }}$ ) mice, as depicted in Figure 7A. Compared with Pten $^{p t K O}$ mice, the Egfr Pten ${ }^{\text {ptDKO }}$ mice exhibited a significantly reduced kidney/BW ratio (Figure $7 \mathrm{~B}$ ); there was no difference in the kidney/BW ratios in any of the 3 controls: Egfr ${ }^{\mathrm{Ctrl}}$ (Egfrflfl) mice, Pten ${ }^{\mathrm{Ctrl}}$ mice, and doublecontrol Egfr Pten ${ }^{\text {DCtrl }}$ mice.

Our signaling experiments revealed that the increased basal levels of Ser473-phosphorylated AKT and Thr389phosphorylated S6K1 observed in Pten ${ }^{\text {ptKO }}$ kidneys were markedly attenuated in the kidneys of Egfr Pten ${ }^{\text {ptDKO }}$ mice

Previous studies demonstrated a concomitant increase in the cell cycle inhibitors $\mathrm{p} 21^{\mathrm{Cip} 1 / \mathrm{WAF}}$ and $\mathrm{p} 27^{\mathrm{Kip} 1}$ during compensatory renal hypertrophy $(34,35)$. Of interest, $\mathrm{p} 21^{\mathrm{Cip} 1 / \mathrm{WAF}}$-positive cells in LTA-positive proximal tubules markedly increased in the enlarged kidneys of Pten ${ }^{p t K O}$ mice compared with that seen in Pten ${ }^{\text {Ctrl }}$ mice

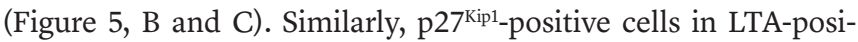
tive proximal tubules also significantly increased in the enlarged Pten $^{\text {ptKo }}$ kidneys (Figure 5, D and E).

Rapamycin had no effect on mTORC2/AKT signaling but inhibited mTORC1/S6K1 signaling and attenuated Pten KO-induced renal growth. Upon entry into mammalian cells, rapamycin forms a complex with the immunophilin FK506-binding protein 12 (FKBP12). This complex subsequently binds to the FKBP12-rapamycin-binding (FRB) domain of mTOR within mTORC1 and inhibits the kinase activity of mTORC1 (36); in contrast, the mTOR in mTORC2, which phosphorylates its downstream effector AKT on Ser473 and thus activates AKT (13), does not bind rapamycin-FKBP12 and has been demonstrated to be rapamycin insensitive (37). Administration of rapamycin (1 $\mathrm{mg} / \mathrm{kg} \mathrm{BW}$, daily by i.p. injection) to 10 -week-old Pten $^{p t K O}$ mice for 7 days indeed had no effect on either mTORC2-
(Figure 7, C and D). These findings collectively link EGFR activity to PTEN/class I PI3K/mTORC2/AKT/mTORC1/S6K1 signaling in the modulation of sizing of renal mass to that of lean body mass.

UNX induced further growth of the remaining kidney in Pten ${ }^{\text {ptKO }}$ mice. Previous studies demonstrated that activation of the mTORC1/S6K1 signaling pathway mediates hypertrophic renal growth in response to contralateral nephrectomy $(22,23)$. Following UNX, 25\% of compensatory renal growth derives from early and brief cellular proliferation and 75\% from subsequent enlargement of tubular and glomerular cells over several weeks (31). As shown in Figure 8, our results revealed that contralateral nephrectomy in $P t e n^{p t K O}$ mice induced further renal hypertrophy in the remaining kidney, as indicated by further increases in kidney size (Figure 8A), kidney/BW ratio (Figure 8B), and protein/DNA ratio (Figure $8 \mathrm{C}$ ), although the degree of incremental compensation was comparable to that seen in WT mice (Figure 8D). Unlike the renal growth of naive Pten $^{p t K O}$ mice, which only displayed enlargement of the proximal tubules but not of the glomeruli (Figure 2, C-F), UNX-induced compensatory hypertrophy in Pten $^{p t K o}$ mice occurred not only in the proximal tubules but also in the glomeruli (Figure 9, A-D). 
A
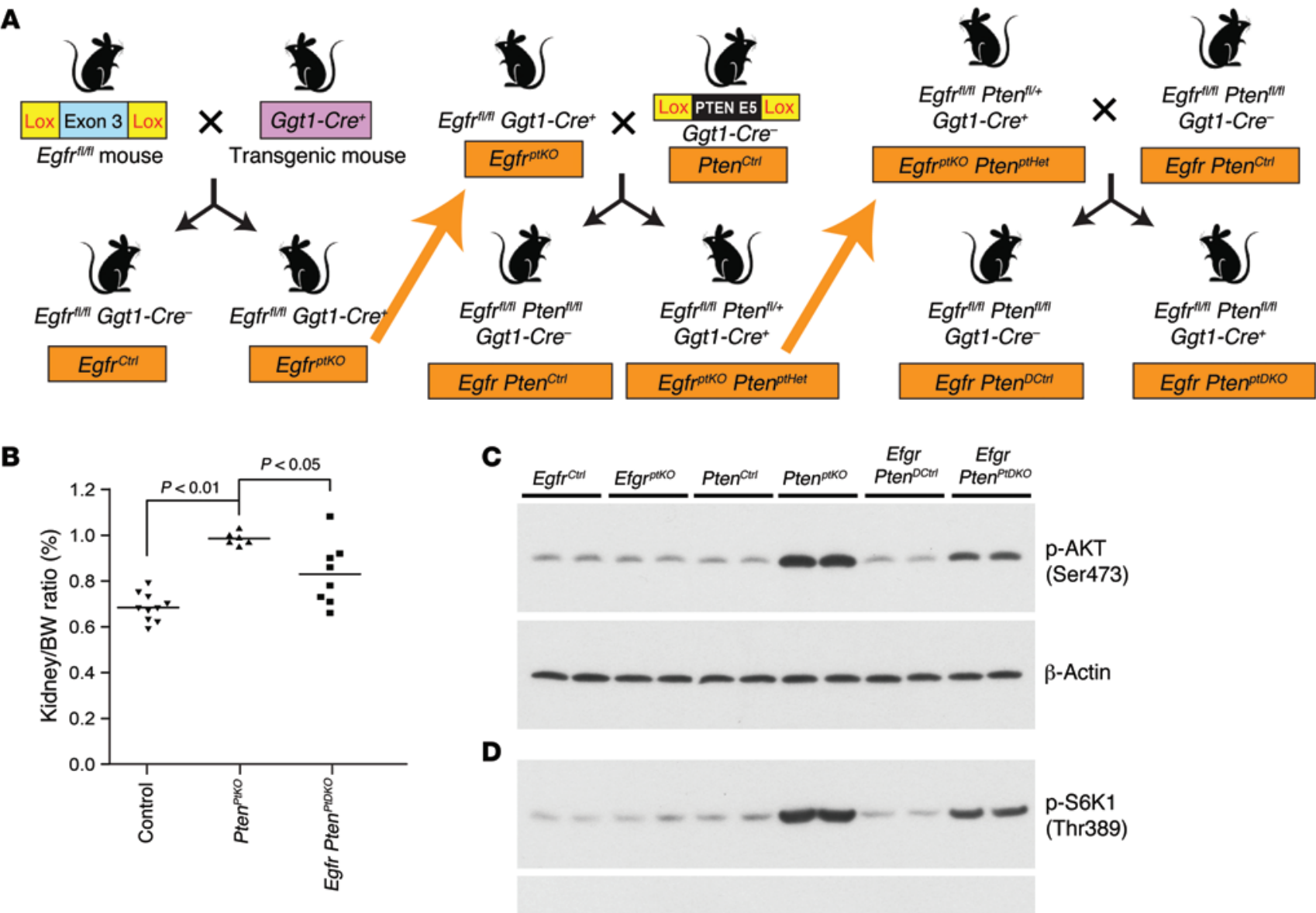

$\beta$-Actin

Figure 7. Simultaneous deletion of proximal tubule EGFR decreases the renal hypertrophy and AKT/mTOR signaling seen in Pten ${ }^{\text {ptKo }}$ mice. (A) Schematic diagram depicting the generation of Egfr and Pten double-KO mice with the Egfrl/ffl Pten fl/fl phenotype (abbreviated as Egfr Pten ${ }^{p t o k o}$ ). Deletion of both Pten and Egfr was confirmed by PCR and immunoblot analysis (data not shown). (B) The increased kidney/BW ratio in Pten $n^{p t K o}$ mice was partially inhibited in Egfr Pten ${ }^{p t o k o}$ mice. ANOVA with Bonferroni's $t$ correction was used for statistical analysis of the data. The indicated $P$ values were calculated from 6 to 10 mice per group. (C and D) The increased levels of AKT (C) and S6K1 (D) seen in the kidneys of Pten ${ }^{\text {ptKo }}$ mice were both partially inhibited in Egfr $P$ ten $^{\text {ptDKo }}$ mice. Shown are representative immunoblots for 6 to 10 mice per group with similar results. There were no differences in kidney/BW ratio among

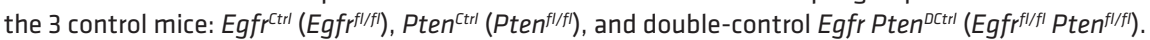

UNX activated $m$ TORC1 but not mTORC2 in the remaining kidney. Neither the basal level of Ser473-phosphorylated AKT and Thr1462-phosphorylated TSC2 in the remaining kidney of WT mice, nor the Pten deletion-elevated AKT and TSC2 phosphorylation in the remaining kidney of Pten ${ }^{\text {ptKO }}$ mice, was augmented by UNX (Figure 10A), indicating that PTEN/mTORC2/ AKT signaling was not involved in the compensatory responses elicited by UNX. As indicated in Figure 10B, following contralateral nephrectomy, the remaining kidneys of both Pten ${ }^{p t K O}$ and WT Pten $^{C t r l}$ mice exhibited markedly higher levels of S6K1 phosphorylation at Thr389, the site directly phosphorylated by MTORC1 for S6K1 activation $(41,42)$. UNX-induced increases in the levels of Ser235/236-phosphorylated rpS6 further confirmed activation of the mTORC1/S6K1 signaling pathway (Figure 10C and refs. 22, 23). Thus, unlike the mechanism mediating Pten deletion-induced kidney growth, mTORC1/S6K1 signaling-driven compensatory renal hypertrophy induced by UNX is independent of the PTEN/ class I PI3K/mTORC2/AKT signaling pathway.
Increased delivery of amino acids stimulated class III PI3K activity and induced hypertrophy in the kidneys of WT mice. Previous studies have indicated that amino acids can activate mTORC1 through a non-class I PI3K/AKT-mediated pathway that involves activation of class III PI3K $(43,44)$. In the kidney, proximal tubular epithelial cells are responsible for the reabsorption of amino acids in the glomerular filtrate. We were able to maintain mouse renal proximal tubular epithelial (MCT) cells in a 1:1 mixture of DMEM and Ham's F-12 culture medium (Invitrogen, Life Technologies), so we defined the concentration of amino acids in this culture medium as $1 \times$. To determine whether increased plasma levels of amino acids will activate mTORC1 in the kidney, 8-week-old inbred male DBA $/ 2$ mice were treated with either vehicle alone or $2 \times$ amino acids, as described in Methods. As shown in Figure 11, compared with vehicle-treated mice, increased delivery of amino acids markedly increased the activity of class III PI3K (Figure 11A) and increased the level of S6K1 phosphorylation at T389, confirming $\mathrm{mTORC} 1$ activation (Figure 11B). 
A
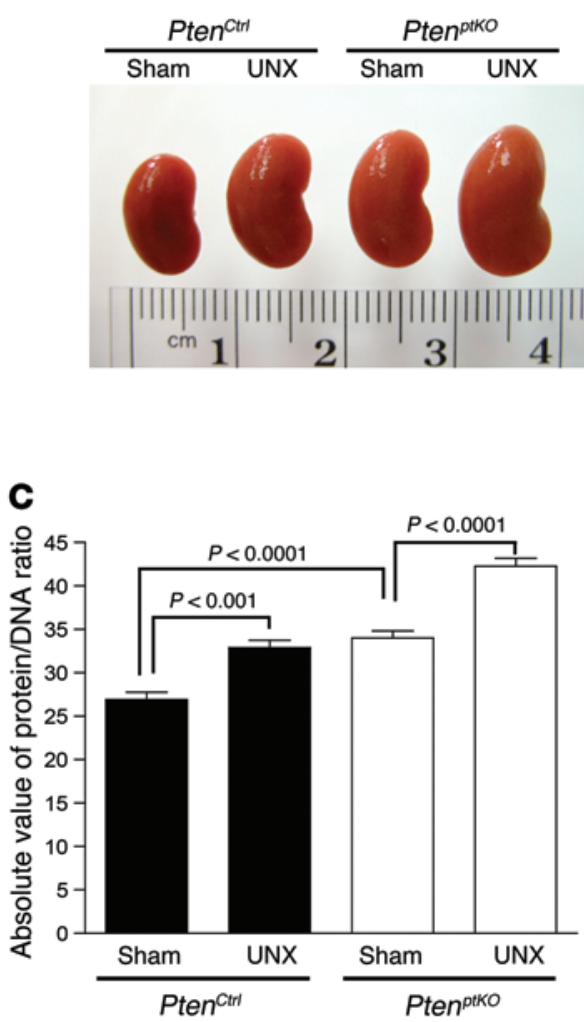

B
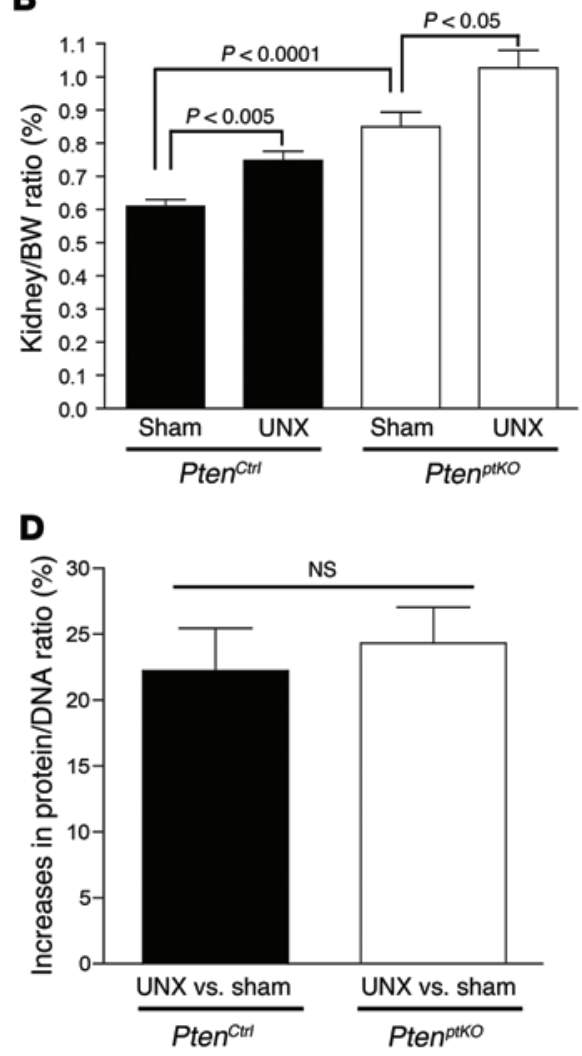

Figure 8. UNX induces equivalent levels of compensatory renal hypertrophy in Pten ptko and $\boldsymbol{P t e n}^{\mathrm{ctrl}}$ mice. Compared with shamoperated Pten ${ }^{p t K o}$ mice and $P$ ten $^{c t r l}$ mice, UNX stimulated equivalent levels of renal hypertrophy in Pten ${ }^{p t K o}$ and Pten ${ }^{\text {ctrl }}$ mice, respectively, as indicated by the increases in kidney size (A), kidney/BW ratio (B), absolute value of protein/DNA ratio (C), and comparable increases in protein/DNA ratio (D) of the remaining kidney. Image in $\mathbf{A}$ shows representative left kidneys from 8 -week-old $P_{t e n^{p t K o}}$ mice and their Pten ${ }^{\complement t r l}$ littermates 4 days after sham surgery or UNX. ANOVA with Bonferroni's $t$ correction was used for statistical analysis of the data in B-D. $n=5-8$ mice per group with similar results. $P$ values are indicated in the respective figures.
Recent studies have documented that amino acids activate mTORC1 by inducing translocation of mTORC1 to the lysosomal membrane where the mTORC1 activator RHEB resides, which activates mTORC1 in association with the RAG GTPase heterodimers and the MP1, p14, and p18 trimeric protein complex termed Ragulator $(45,46)$. Of note, previous studies have also demonstrated that the population of mTOR at the lysosomal membrane represents mTORC1, as the colocalization of mTOR with lysosome-associated membrane protein (LAMP) 1 or 2 is lost upon knockdown of the mTORC1-defining component RAPTOR $(20,46)$. Within 30 minutes of amino acid infusion, we observed increased colocalization of MTOR at the lysosomal membrane with LAMP1, a well-established marker specific for lysosomal membranes (47), in the kidneys of WT mice (Figure 11C). Moreover, chronically increasing delivery of amino acids to WT mice via their drinking water for 2 weeks caused significant increases in the renal protein/DNA ratio (Figure 11D) and the kidney/BW ratio (Figure 11E), indicating increased protein synthesis and renal hypertrophy.

Amino acid administration has been demonstrated to stimulate protein synthesis $(48,49)$ and induce rpS6 phosphorylation in a rapamycin-sensitive manner $(50,51)$. Using insulin as a positive control for mTORC1 activation by the class I PI3K/AKT pathway $(16,21)$, we found that in cultured renal proximal tubule cells, amino acids did not induce an increase in Ser473-phosphorylated AKT levels (Figure 12A), but stimulated a marked increase in Thr389-phosphorylated S6K1 levels (Figure 12B). As shown in Figure 12C, immunoprecipitation with an antibody specifically recognizing class III PI3K, followed by in vitro kinase activity assays using phosphatidylinositol (PI) as a substrate, revealed that amino acids, but not insulin, activated the kinase activity of class III PI3K in renal proximal tubule cells. Furthermore, siRNA-mediated downregulation of class III PI3K protein expression (Figure 12A) inhibited amino acid-induced S6K1 phosphorylation at Thr389 without affecting the stimulation by insulin (Figure 12B), with a consistent inhibition of the lipid kinase activity of class III PI3K (Figure 12C). As indicated in Methods, these results were further confirmed by using 2 additional sets of individual siRNAs that were previously demonstrated to be effective and specific for knocking down class III PI3K (data not shown) (43). This selective mTORC1 activation by amino acids in the cultured proximal tubule cells is consistent with previous demonstrations of such a role for class III PI3K in other cell types $(43,44)$.

As shown in Figure 12D, quiescent renal proximal tubular epithelial (MCT) cells deprived of serum and amino acids had minimal mTOR localization on the lysosomal membranes, as evidenced by the minimal mTOR colocalization with LAMP1. Of interest, readdition of amino acids to the quiescent cells markedly induced mTOR colocalization with LAMP1 (Figure 12D), but siRNA-mediated ablation of class III PI3K prevented amino acids from inducing mTOR colocalization with LAMP1 (Figure 12E). These results further indicate that class III PI3K plays an essential role in mediating amino acid-induced mTORC1 activation through a mechanism involving the recruitment of mTOR to its activation site - the lysosomal membranes $(45,46)$.

UNX increased the activity of class III PI3K and induced mTOR localization on the lysosomal membrane in kidney. Previous studies have shown that upon removal of 1 kidney, renal blood flow 
A

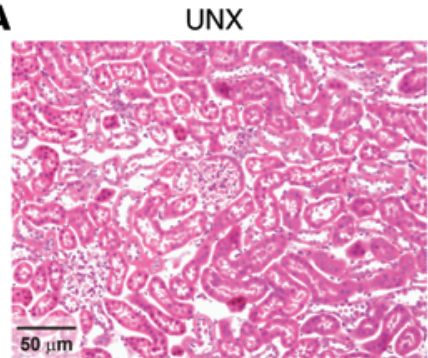

Pten ${ }^{\text {Ctr }}$

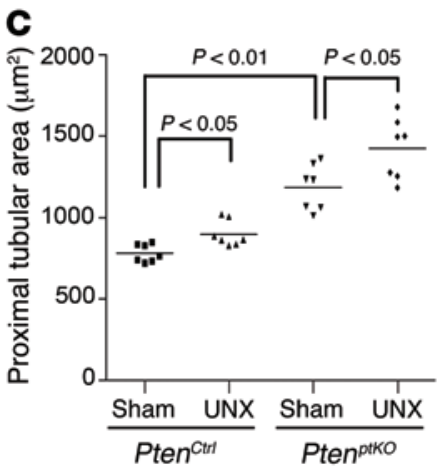

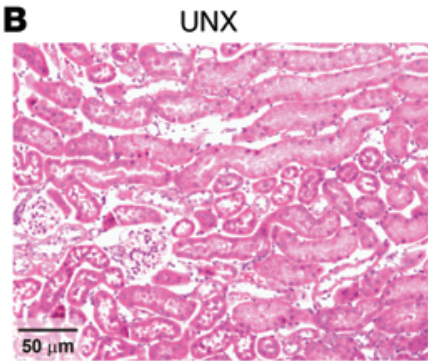

Pten ptKo

D

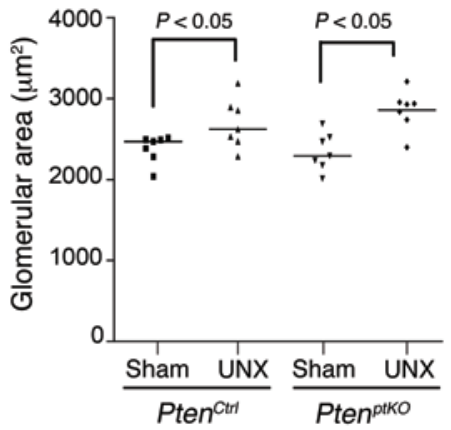

Figure 9. UNX induces both renal proximal tubular and glomerular enlargement in Pten ${ }^{\text {ptKo }}$ as well as Pten ${ }^{\text {Ctr }}$ mice. (A and B) UNXinduced hypertrophy occurred not only in the proximal tubules but also in the glomeruli in both Pten $^{\text {ctrl }}$ (compare $\mathbf{A}$ with Figure $2 \mathrm{C}$ ) and Pten ${ }^{\text {ttKo }}$ mice (compare B with Figure 2D). (C and D) Quantification by morphometric analysis of proximal tubule area and glomerular area in sham-operated or UNX-operated Pten ${ }^{c t r l}$ and $P t e n^{p t K o}$ mice revealed that, unlike the renal growth in sham-operated $P t_{t e n}{ }^{p t K o}$ mice, which did not differ from that seen in the naive $P$ ten $^{\text {ptKo }}$ mice that only exhibited enlargement of the proximal tubules but not of the glomeruli (also revealed in Figure 2, C-F), UNX-induced compensatory hypertrophy occurred not only in the proximal tubules but also in the glomeruli of both Pten ${ }^{\text {ctrl }}$ and Pten ${ }^{p t K o}$ mice (C and $\left.\mathbf{D}\right)$. Shown in $\mathbf{A}$ and B are representative images from 8-week-old $P$ ten $^{\text {ctrl }}$ and $P$ ten $^{\text {ptKo }}$ mice 4 days after right UNX. ANOVA with Bonferroni's $t$ correction was used for statistical analysis of the data in $\mathbf{C}$ and $\mathbf{D}$. $n=7$ mice per group with similar results. $P$ values are shown in the figures to indicate specific comparisons between groups. Scale bars: $50 \mu \mathrm{m}$.
$(\mathrm{RBF})$ to the remaining kidney increases immediately, with a $16 \%$ increase still being seen after 28 days (52). Using an OxyFlow system, we also observed an immediate increase in RBF in response to ligation of the contralateral renal pedicle even before the removal of the kidney; this RBF signal decreased to zero following sacrifice of the mice by isoflurane overdose (Figure 13A). By reverse-phase HPLC analysis, we also observed that UNX induced a marked increase in the concentration of free amino acids in the remaining kidney within 3 minutes, followed by a slightly lessened but persistent increase even after 24 hours (Figure 13B). We also observed markedly increased lipid kinase activity of class III PI3K in the remaining kidney in response to contralateral nephrectomy (Figure 13C).

UNX stimulated markedly increased mTOR colocalization with LAMP1 in the tubular epithelial cells of the remaining kidney compared with that seen in sham-operated mice, indicating that mTOR was markedly accumulated on the lysosomal membranes of renal tubular epithelial cells in response to removal of the contralateral kidney (Figure 13D). Thus, our observations support the concept that increased delivery of amino acids into the remaining kidney by increased RBF in response to contralateral nephrectomy is an important initial growth signal that activates class III PI3K to act as an upstream mediator for activation of the mTORC1/S6K1 signaling pathway, which underlies compensatory renal hypertrophy following loss of renal mass (22-24).

\section{Discussion}

In the present study, we first examined the role of PTEN in the regulation of postembryonic kidney size by deleting Pten selectively in renal proximal tubules, which make up the bulk of kidney mass, using a Ggt1 promoter-driven Cre mouse line to cross with Pten ${ }^{f / f l}$ mice. The resulting kidney hypertrophy in these proximal tubulespecific Pten-KO (Pten $\left.{ }^{p t K o}\right)$ mice indicates a previously unappreci- ated and striking inhibitory role for PTEN in controlling kidney size. We reasoned that an upstream positive signal is required for activation of class I PI3K-dependent dynamic PIP3 production $(8,9)$ and identified EGFR as a positive regulator upstream of class I PI3K activation, because when we deleted EGFR in the renal proximal tubules of Pten $^{p t K O}$ mice by generating proximal tubule-specific Egfr and Pten double-KO (Egfr Pten ${ }^{\text {ptDKO}}$ ) mice, we observed a marked reduction in Pten KO-induced renal growth. These results demonstrate that EGFR is an important upstream regulator that positively maintains the dynamic activity of the class I PI3K/mTORC2/AKT signaling pathway, while PTEN negatively regulates such signaling activities. Thus, our studies indicate that such a balanced counterregulation is an important mechanism underlying the tightly maintained normal kidney size relative to body mass.

Our studies also revealed that in the context of UNXinduced kidney growth, the mTORC2/AKT signaling pathway is not activated. Therefore, additional experiments were directed at identifying the long-sought-after initial signal that activates the mTORC1/S6K1 signaling pathway mediating UNX-induced compensatory renal hypertrophy $(22,23)$. We observed an immediate increase in $\mathrm{RBF}$ in response to ligation of the contralateral renal pedicle. We also observed a marked increase in the concentration of free amino acids in the remaining kidney following removal of the contralateral kidney, and our in vitro cell culture studies revealed that class III PI3K mediates the activation of mTORC1/ S6 K1 signaling in renal proximal tubular epithelial cells in response to amino acid stimulation. Amino acids activated the kinase effect of class III PI3K, and siRNA-mediated downregulation of class III PI3K expression inhibited amino acid-induced increases in S6K1 phosphorylation at Thr389, the site phosphorylated by mTORC1 kinase activity. Our in vivo animal studies revealed that the kinase activity of class III PI3K was markedly increased in the remaining kidney in response to contralateral nephrectomy. 


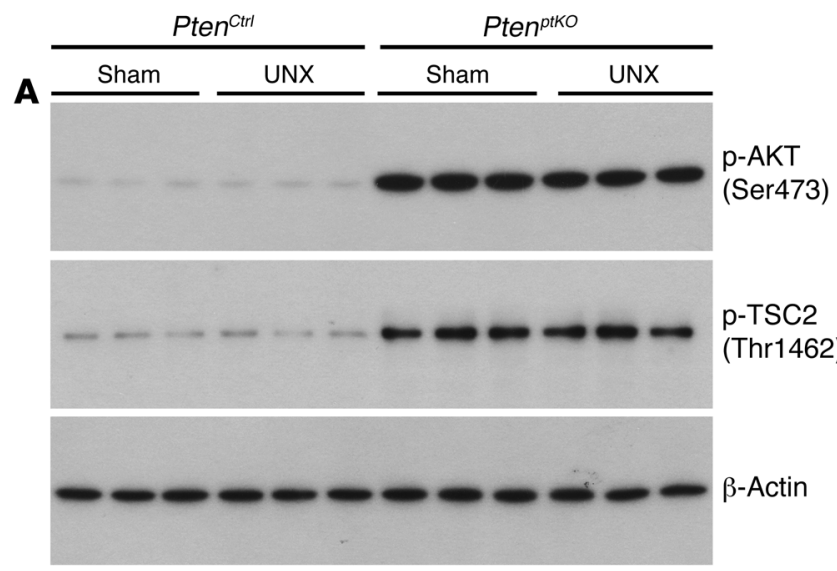

B

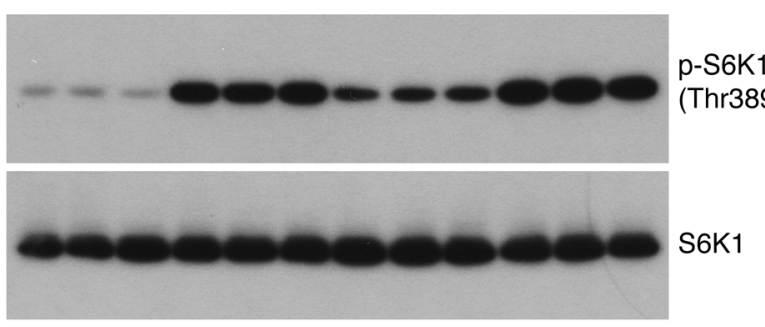

C

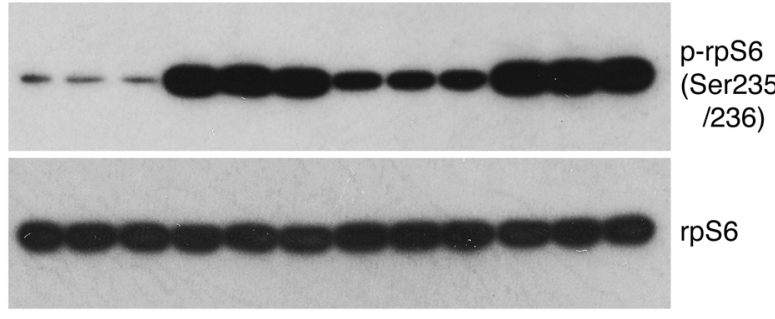

Figure 10. UNX activates mTORC1 signaling but not AKT signaling in the remaining kidney. Compared with sham operation, UNX had no effect on AKT or TSC2 phosphorylation (A), but markedly increased the phosphorylation levels of both S6K1 (B) and rpS6 (C) in the remaining kidney in either Pten $^{\text {ptKo }}$ or Pten ${ }^{\text {Ctrl }}$ mice, respectively. Shown are representative immunoblots for 7 mice per group with similar results.

Our further experiments confirmed that increased delivery of exogenous amino acids markedly increased the activity of class III PI3K, induced translocation of mTOR to the lysosomal membrane, and activated mTORC1 signaling to S6K1 phosphorylation at Thr389, leading to increases in both protein/DNA and kidney/BW ratios (indicating renal hypertrophy) in WT mice, independent of any KOs. This finding is consistent with previous studies indicating that a high-protein diet can induce kidney hypertrophy (53). Moreover, our additional experiments demonstrated greater mechanistic insight into how amino acids activate mTORC1 in renal proximal tubule cells. Specifically, our in vitro studies suggest that amino acids stimulate mTORC1 activation by inducing class III PI3K-dependent translocation of mTOR to the lysosomal membrane, which is the site of mTORC1 activation $(45,46)$, in cultured renal proximal tubular cells. We also confirmed that UNX increased accumulation of mTOR on the lysosomal membrane in the renal tubular cells in the remaining kidney in WT mice.
Our previous results had demonstrated that activation of mTOR-dependent signaling was a necessary component of contralateral nephrectomy-mediated compensatory renal hypertrophy (22-24). Our new results suggest that increased amino acid delivery to the remaining kidney due to increased RBF is an early signal that induces activation of class III PI3K and translocation of mTOR to the lysosomes, leading to activation of mTORC1 signaling to phosphorylation of S6K1 and, consequently, activation of the S6K1/rpS6 signaling pathway for the initiation of increased protein synthesis and cell growth, which underlie compensatory kidney hypertrophy (depicted in Figure 14). We have attempted to further confirm the involvement of class III PI3K in UNX-induced renal hypertrophy in a $\mathrm{KO}$ mouse model by deleting the mouse class III PI3K gene. However, germline deletion of class III PI3K by breeding $P i k 3 c 3^{f / f l}$ mice with a CAG-Cre-driver strain (The Jackson Laboratory), deleting the gene at the 1-cell embryo stage, resulted in embryonic lethality, and even crossing $P i k 3 c 3^{f / f l}$ mice (54) with Ggt1-Cre mice (28) or Pepck-Cre mice (55) did not result in viable class III PI3K-KO mice.

The phenomenon of compensatory renal hypertrophy was first described more than a century ago (56). Compensatory renal hypertrophy is an important consequence in remaining renal tissue following partial or complete UNX as well as in both recipients and living donors following renal transplantation. In addition, it is recognized that compensatory renal hypertrophy occurs in virtually all kidney diseases that cause nephron damage and, consequently, a reduction in the number of functioning nephrons (57-60). Evidence suggests that excessive compensatory renal hypertrophy may sometimes be a maladaptive response that sets the stage for the inexorable progression of further nephron damage, interstitial fibrosis, tubular atrophy, progressive decline of renal function, and development of end-stage renal disease, while attenuation of compensatory renal hypertrophy might limit progressive kidney damage (56-59). However, the underlying signals and mechanisms mediating both the normal regulation of kidney size and the onset and extent of compensatory renal hypertrophy have long been a mystery. Class III PI3K is also known as the mammalian homolog of yeast vacuolar protein sorting defective 34 (mVps34). Although Vps34 is the only PI3K in yeast (61), mammals have evolved to express 3 different classes of PI3Ks (14), and our studies here reveal that both class I and class III PI3K signaling activities are important mediators of the tight regulation of kidney size in a context-dependent manner to maintain a relatively constant kidney mass/lean body mass ratio so that the homeostasis of a healthy body can be achieved. These findings could lead to therapeutic strategies for preventing progressive nephron damage by judicious pharmacological modulation of class I and/or class III PI3K signaling activities, as virtually all kidney diseases that cause nephron damage could ultimately lead to maladaptive hypertrophy in the residual surviving nephrons.

\section{Methods}

Reagents and antibodies. Most reagents, including $\beta$-actin monoclonal antibodies, were purchased from Sigma-Aldrich. Rapamycin was purchased from LC Laboratories. Antibodies against PTEN (catalog 9552), p-AKT (Ser473; catalog 4060), p-AKT (Thr308; catalog 2965), p-tuberin/TSC2 (catalog 3617), p70 S6 kinase (catalog 2708), p-p70 


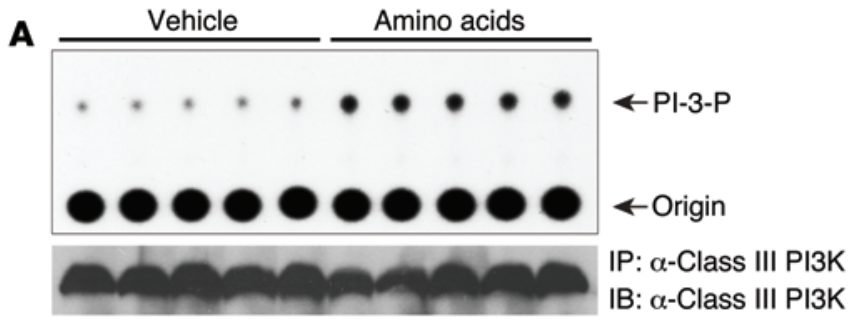

B

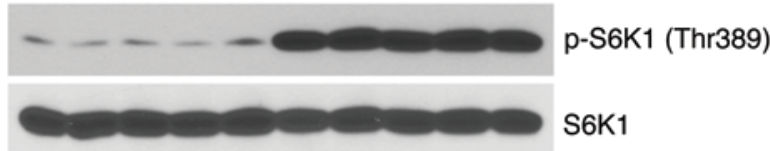

C
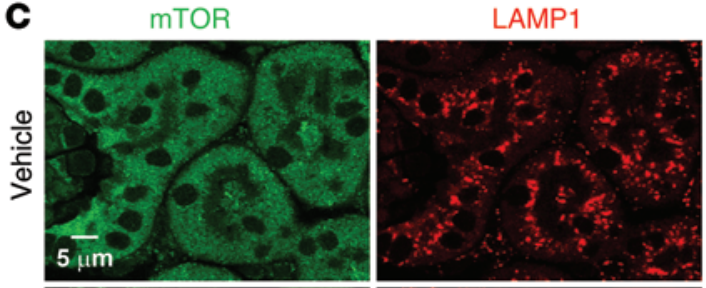

MTOR/LAMP1/DAPI
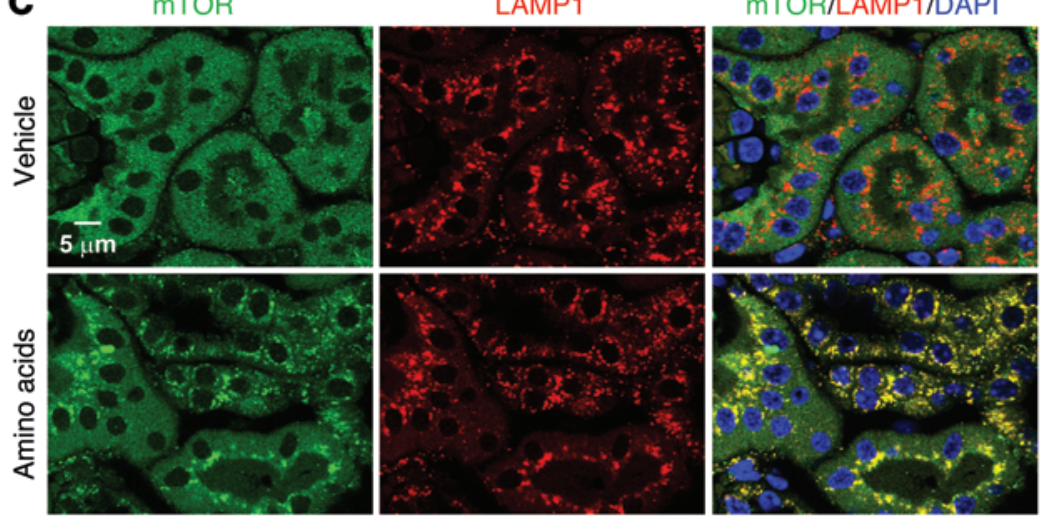

D
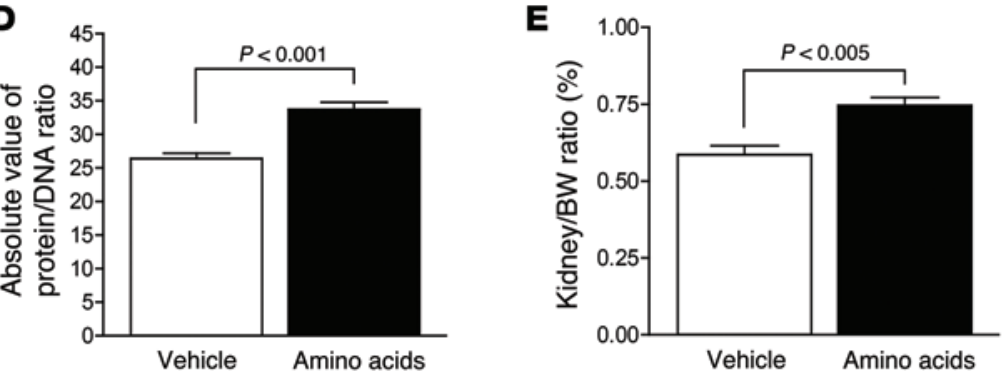

Figure 11. Increased delivery of amino acids increases class III PI3K activity, induces mTOR translocation to lysosomal membranes and activation, and induces hypertrophy in the kidneys of WT mice. Inbred 8-weekold male DBA/2 mice were injected through the tail vein with either $2 \times$ amino acids or vehicle (saline) control as detailed in Methods. Thirty minutes later, the mice were sacrificed and kidney samples harvested to measure class III PI3K activity by immunoprecipitation with anti-class III PI3K antibodies, followed by an in vitro lipid kinase activity assay using PI as a substrate (A, top); an aliquot of the immunoprecipitated proteins (IP) was subjected to immunoblotting (IB) with an anti-class III PI3K antibody to detect the amounts of immunoprecipitated class III PI3K (A, bottom). The remaining harvested kidney samples were used to measure mTORC1 signaling to S6K1 phosphorylation at Thr389 (B), and confocal microscopy was used to detect the localization of mTOR (green) and LAMP1 (red), with nuclei highlighted by DAPI (blue) (C). (D and E) Inbred 8-week-old male DBA/2 mice were given daily fresh $4 \times$ amino acids through the drinking water $(13.3 \mathrm{ml} 150 \times$ stock amino acids in $500 \mathrm{ml}$ drinking water) or vehicle control (13.3 $\mathrm{ml}$ saline in $500 \mathrm{ml}$ drinking water). Two weeks later, the mice were sacrificed to measure renal protein/ DNA (D) and kidney/BW (E) ratios. A 1-tailed, unpaired $t$ test was used for statistical analysis of the data in $\mathbf{D}$ and E. $n=5$ mice per group. $P$ values are shown in the figures to indicate specific comparisons.
S6K1 (catalog 9234), rpS6 (catalog 2217), and p-rpS6 (catalog 4856) were purchased from Cell Signaling Technology. Antibodies against THP glycoprotein (catalog 55140) were obtained from MP Biomedicals. Ki67 antibody (catalog NCL-Ki67p) was purchased from Novocastra Laboratories. Collagen type I antibody (catalog AB765P) was purchased from EMD Millipore. Class III PI3K antibodies (catalog 38-2100) were purchased from Zymed Laboratories (Life Technologies). Antibodies against p21 (catalog sc6246), p27 (catalog sc528), and LAMP1 (catalog sc-19992) were purchased from Santa Cruz Biotechnology Inc. $\left[\gamma^{-32} \mathrm{P}\right]$ ATP (specific activity, $3,000 \mathrm{Ci} / \mathrm{mmol}$ ) was obtained from PerkinElmer Life and Analytical Sciences LLC. PI was obtained from Avanti Polar Lipids Inc. Biotin-conjugated LTA (catalog B-1325), FITC-conjugated LTA (catalog FL1321), biotin-conjugated DBA (catalog B-1035), and biotin-conjugated anti-rabbit IgG antibody (catalog BA-1000) were purchased from Vector Laboratories. Cy3-conjugated anti-goat antibody (catalog 705-165-003) was purchased from Jackson ImmunoResearch Laboratories. Alexa Fluor 555 (catalog S-21381) or Alexa Fluor 647-conjugated streptavidin (catalog S-21374) was purchased from Molecular Probes.
Animals, surgical procedures, and rapamycin treatment. Animals were housed at either the Vanderbilt University veterinary facility (MRBIII) or the Georgia Regents University veterinary facility (CA Building) for the different experiments performed in the present study. Pten ${ }^{f / f l}$ mice on a BALB/cByJ background were purchased from the The Jackson Laboratory. Inbred male DBA/2 mice were from Taconic. We have previously described the generation of the Ggt1-Cre transgenic mouse line (28); this Ggt1-Cre mouse line was backcrossed with BALB/cByJ for 7 generations before mating with the $P t e n^{f / f l}$ mice to generate renal proximal tubule cell-specific homozygous Pten-KO (Pten ${ }^{p t K O}$ ) mice. Compensatory renal hypertrophy was induced as we previously described (22-24). For experiments testing the effect of rapamycin, mice were administered either rapamycin (1 mg/kg BW, daily by i.p. injection) or vehicle alone for 7 days. For studies examining the role of EGFR, we used Egfrf/ll mice on a BALB/cByJ background. These mice were crossed with Gtgl-Cre mice (28) to produce proximal tubule-specific Egfr-KO (EgfrptKo mice (40), and then further crossed with $P$ ten $^{f / f l}$ mice to produce renal proximal tubule-specific Egfr and Pten double-KO (Egfr Pten $\left.{ }^{p t D K O}\right)$ mice and double-control (Egfr Pten ${ }^{\text {DCtr }}$ ) mice, as depicted in Figure 7A. 
A

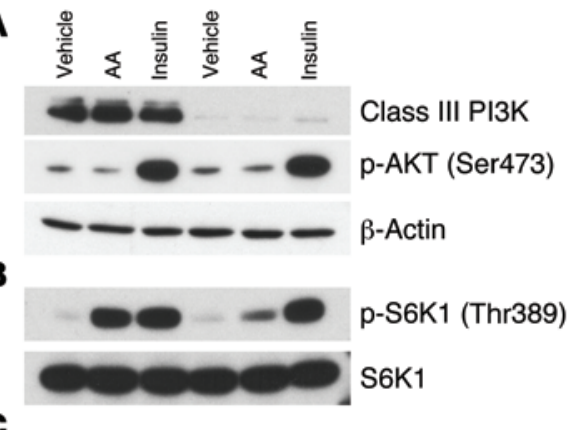

C

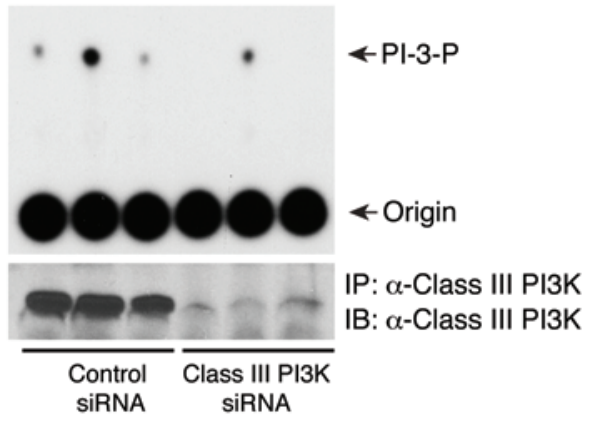

D
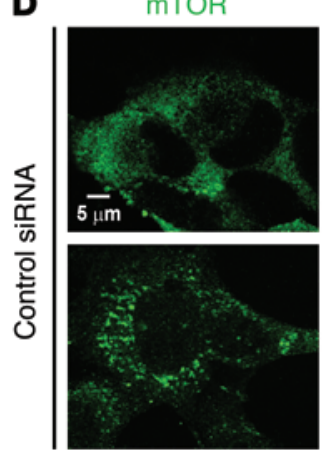

E
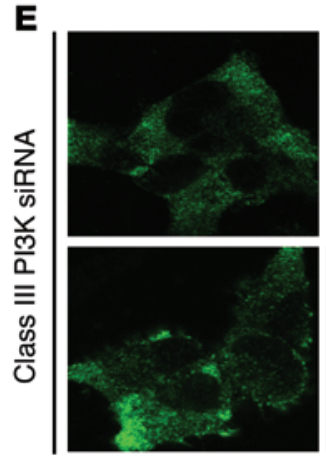

LAMP1
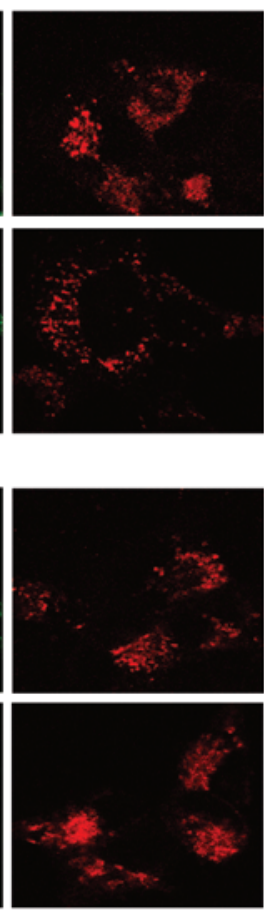

mTOR LAMP1 DAPI
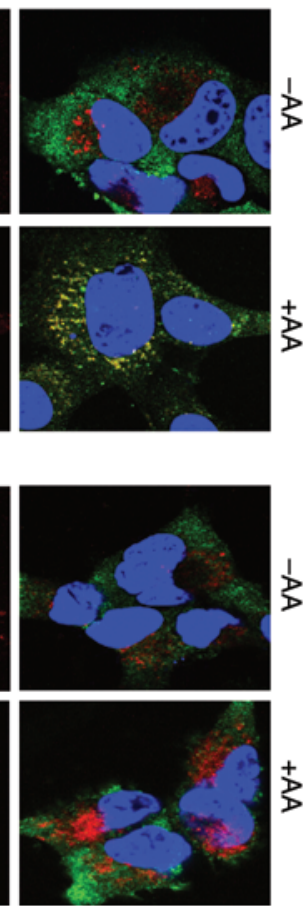

Figure 12. Knockdown of class III PI3K activity inhibits mTOR translocation to lysosomal membranes and activation in cultured renal proximal tubule epithelial cells. (A-C) Mouse MCT cells transfected with either scrambled control siRNA or class III PI3K-specific siRNA and made quiescent before treatment with $1 \times$ amino acids, $100 \mathrm{nM}$ insulin, or vehicle (saline) alone for 30 minutes. Cell lysates were subjected to immunoblotting (A and B) and class III PI3K activity assays (C, top), along with confirmation of the levels of immunoprecipitated class III PI3K (C, bottom) by immunoblotting following immunoprecipitation with anti-class III PI3K antibodies. (D and E) MCT cells were transfected with scrambled control siRNA (D) or siRNA specific for mouse class III PI3K (E). Cells were made quiescent 48 hours after transfection as detailed in Methods and then restimulated with vehicle control (-AA) or $1 \times$ amino acids (+AA) for 10 minutes. Cells were then processed for coimmunofluorescence staining to detect endogenous mTOR (green) and the lysosomal membrane marker LAMP1 (red), along with DAPI to highlight nuclei (blue), and imaged by confocal microscopy. The mTOR and LAMP1 localization pattern was exhibited by $90 \%$ to $100 \%$ of the cells. Shown are representative images from at least 3 independent cell culture experiments with similar results. Scale bar: $5 \mu \mathrm{m}$.

Treatment of WT mice with increased delivery of amino acids. Inbred male DBA/2 mice (Taconic) were randomly divided at 8 weeks of age into amino acid treatment groups and vehicle treatment groups (5 mice per group). For acute early signaling studies, we directly injected $2 \times$ amino acids into the tail vein of mice in 1 amino acid treatment group. $1 \times$ is defined as the concentration of amino acids present in the nutrient-enriched DMEM/F-12 cell culture medium, which was a 1:1 mixture of DMEM and Ham's F-12 (Invitrogen, Life Technologies). Given that, on average, the mice have $58.5 \mu$ of blood per gram of BW, we injected $9 \mu 15 \times$ stock amino acids per gram of BW to ensure all the mice in the amino acid treatment group had exactly the same degree of increased delivery of amino acids, attempting to achieve a final concentration of $2 \times$ amino acids. All the mice in one of the vehicle treatment groups were tail-vein injected with the same amount of vehicle $(9 \mu \mathrm{l}$ saline per gram of BW). Thirty minutes later, the mice were sacrificed to measure class III PI3K activity, mTOR localization, and mTORC1 signaling activity in the kidney.

Since, on average, mice drink $0.15 \mathrm{ml} / \mathrm{g}$ BW/day (usually 3-5 ml water/day) and have $58.5 \mu \mathrm{l}$ of blood per gram of BW, we sought to examine whether chronically increased delivery of amino acids could cause increased protein synthesis leading to renal hypertrophy by providing an additional group of mice with daily fresh $4 \times$ amino acids in the drinking water $(13.3 \mathrm{ml} 150 \times$ stock amino acids in $500 \mathrm{ml}$ drinking water), while another vehicle control group was given drinking water containing the same amount of vehicle $(13.3 \mathrm{ml}$ saline in $500 \mathrm{ml}$ drinking water). Two weeks later, the mice were sacrificed to quantitate protein/DNA ratios in the kidney and kidney/BW ratios.

Genotyping. DNA was extracted and PCR was performed as we have described previously $(28,40)$. The PCR primers used for genotyping were: Pten 5A (TGATAGAACGgAagtCTTGC) and Pten 3C (CATGAGATTAATCACTCACAG) for detection of the Pten-floxed allele; Pten 5A (above) in combination with Pten P3 (GCTTGATATCGAATTCCTGCAGC) (27) for verification of Cre-mediated Pten deletion in the kidney; and Cre 5' (AGGTGTAGAGAAGGCACTTAGC) and Cre 3' (CTAATCGCCATCTTCCAGCAGG) for further confirmation of Ggt1-Cre-positive mice.

Immunofluorescence staining and immunoblot analysis. In addition to standard H\&E staining, paraffin-embedded kidney sections (3 $\mu \mathrm{m}$ in thickness) were also subjected to immunofluorescence staining procedures essentially as we have previously described (24, $28,40)$. Immunoblotting procedures were performed as described previously $(22-24,62,63)$.

Measurement of protein/DNA ratios. Renal cortex and medulla, excluding papilla (0.08 g per sample), were homogenized in a $1.5-\mathrm{ml}$ lysis buffer containing $0.02 \%$ SDS, $150 \mathrm{mM} \mathrm{NaCl}$, and $15 \mathrm{mM} \mathrm{Na}$ 
A

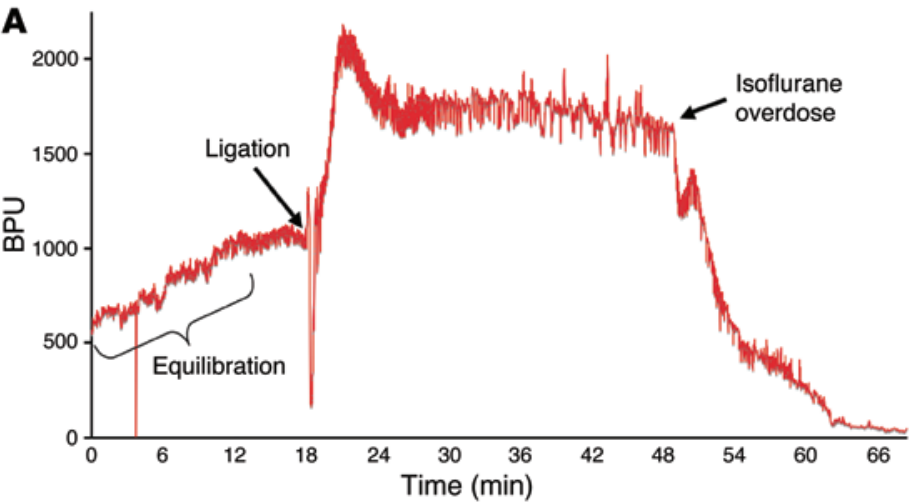

B

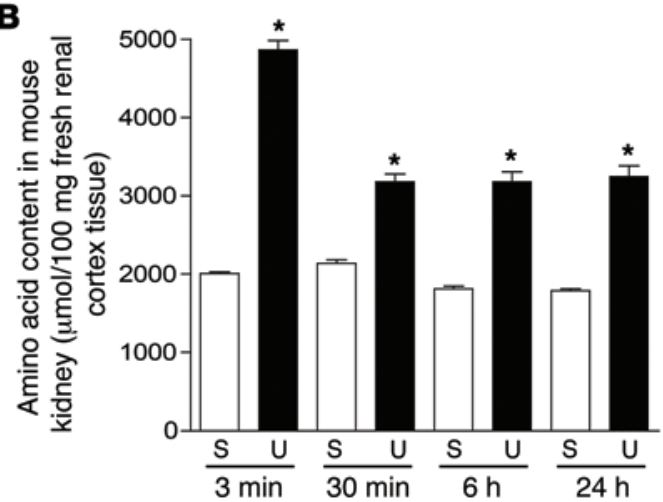

C

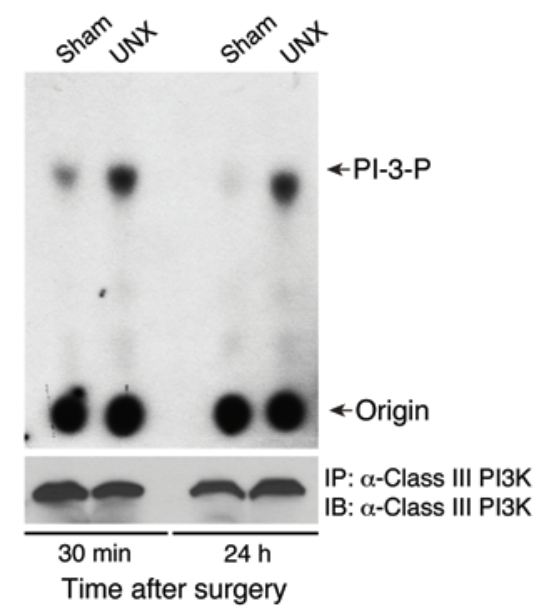

D
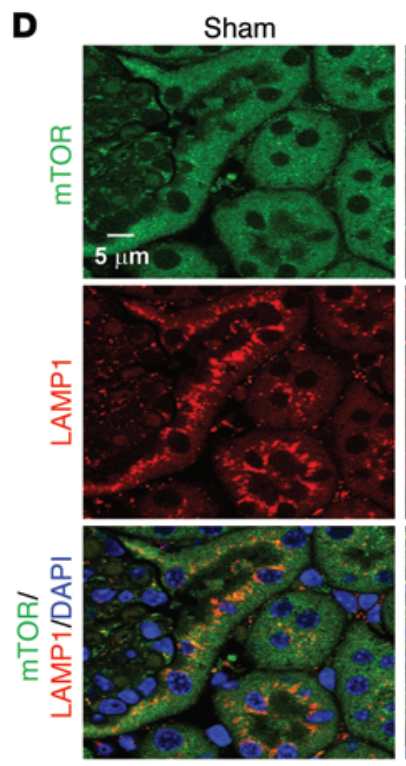
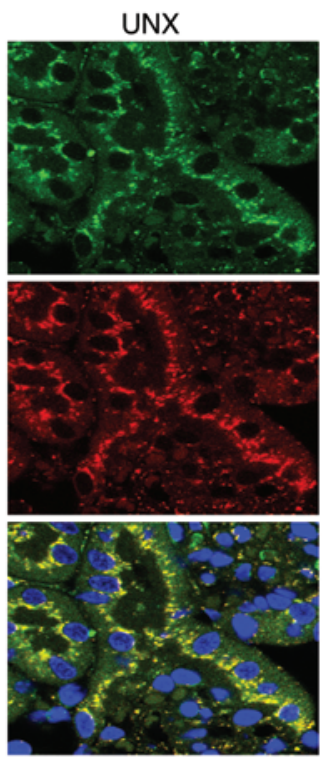

Figure 13. UNX induces increased RBF and renal delivery of amino acids, activates class III PI3K, and induces mTOR localization on the lysosomal membrane in the remaining kidney. (A) An immediate increase in RBF in mice occurred upon ligation of the contralateral renal pedicle, followed by gradually decreased RBF initiated by an overdose perfusion of isoflurane. RBF is expressed as blood perfusion units (BPU). (B) Significant increases were observed in free amino acid content in the remaining kidney of mice in response to UNX (U) compared with sham-operated mice (S). ANOVA with Bonferroni's $t$ correction was used for statistical analysis of the data. ${ }^{*} P<0.001$ indicates a comparison between group $U$ versus group $S$ at each time point, respectively. No significant difference was seen in the $S$ group at different time points $(P>0.05) . n=5$ mice per group per time point. (C) Class III PI3K kinase activity in the left kidney was measured using the same lipid kinase activity assay as in Figure 11A after 8-week-old male DBA/2 mice were subjected to right sham or UNX surgery for the indicated time periods, with the lower panel showing immunoprecipitated class III PI3K. (D) Eight-week-old male DBA/2 mice were subjected to right sham or UNX surgery. The mice were sacrificed 6 hours later to harvest and process the left kidney for immunofluorescence staining and confocal microscopy to detect the localization of mTOR and LAMP1. $n=5$ mice per treatment group for animal experiments with similar results. Scale bar: $5 \mu$ m.

citrate, followed by a 10 -fold dilution. DNA determination was performed in triplicate as we described previously (22-24).

Measurement of cross-sectional cortical tubule area and glomerular surface area. For each mouse, the area of 50 cross-sectional cortical tubules and glomeruli was randomly measured in the H\&Estained sections by the BIOQUANT True-Color Windows System (R \& M Biometrics Inc.) (40).

Quantification of Ki67-, p21-, and p27-positive tubule nuclei. Six photographs were taken randomly at $\times 200$ magnification. The values were calculated by the ratio of Ki67-, p21-, and p27-positive tubule nuclei to the total number of LTA-positive renal proximal tubule cells costained with DAPI to highlight all nuclei (63).

Measurement of amino acids. Male DBA/2 mice, aged 6 weeks, were purchased from Taconic and allowed to adapt to our insti- tutional animal housing environment for 2 weeks. Mice were then subjected to sham or right UNX as described in the surgical procedures above. The mice were sacrificed after surgery at the times indicated above, and the left kidneys were harvested. Renal cortical homogenates were prepared by sonication in the presence of $50 \mu \mathrm{M}$ $\alpha$-aminobutyric acid (as an internal standard) to release free amino acids. After adding equal volumes of $4 \%$ perchloric acid, the homogenates were incubated on ice for 1 hour to precipitate proteins. The renal cortical homogenates were clarified at 20,800 $g$ for 5 minutes at $4^{\circ} \mathrm{C}$. Free amino acid concentrations in the supernatants were determined by reverse-phase HPLC after precolumn derivatization with phenylisothiocyanate as reported previously (64). Amino acid concentrations are expressed as the mean \pm SEM in $\mu$ moles per $100 \mathrm{mg}$ fresh renal cortex tissues. 


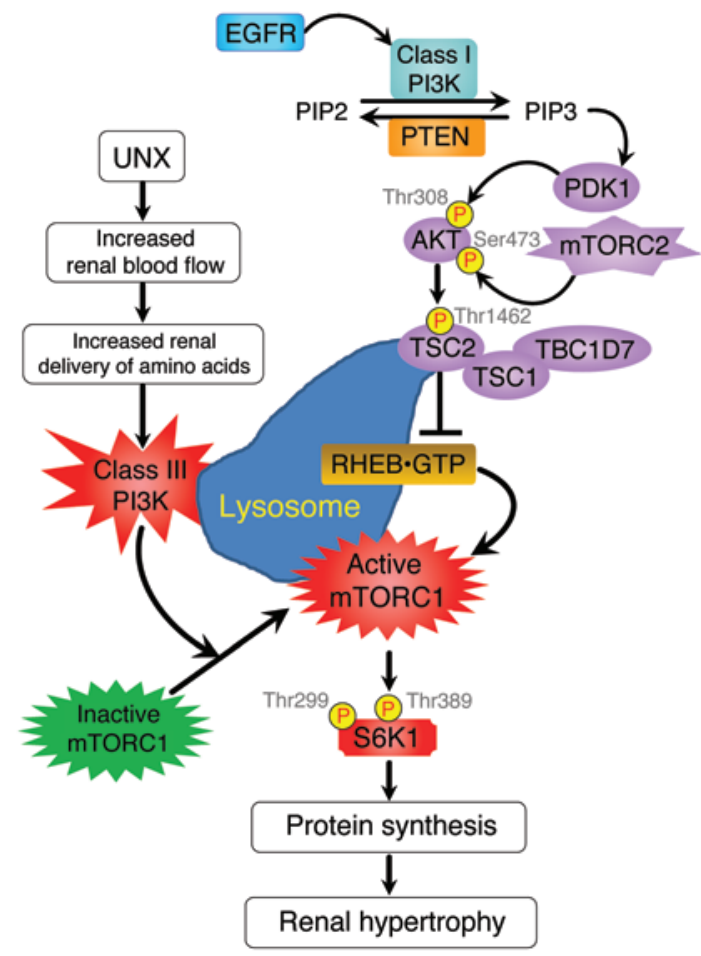

Measurement of RBF. Eight-week-old male DBA/2 mice were used for the measurement of RBF. Briefly, mice were placed on a warming table and anesthetized with constant perfusion of isoflurane. Under aseptic conditions, a right flank incision was made and the right renal pedicle isolated. A loose ligature (4-0 silk suture) was placed around the renal pedicle to ensure that RBF to the right kidney was not occluded, and the right kidney was returned to the retroperitoneum. The left kidney was exposed through the site of a left flank incision, a laser Doppler fiber optic flow probe was inserted, and relative RBF into the left kidney was measured using an OxyFlow system (Oxford Optronix).

Saline-saturated sponges were used to cover up the left kidney to prevent evaporation. After a 15 -minute equilibration period, left RBF was stabilized, and a ligature placed around the right renal pedicle was tightened to completely block RBF to the right kidney. RBF into the left kidney was recorded. After an additional 30 minutes, an overdose perfusion of isoflurane was applied to the mice, and the recording was continued until the RBF dropped to zero as a result of the isoflurane overdose-induced sacrifice of the mice.

Cell culture and treatment. MCT cells, a well-established murine renal proximal tubule cell line that maintains the characteristics of renal proximal tubule cells (65), were maintained in low glucose (5.5 $\mathrm{mM}$ ) Gibco DMEM/F-12 Nutrient Mixture (DMEM/F-12) medium supplemented with 100 units $/ \mathrm{ml}$ penicillin, $100 \mathrm{mg} / \mathrm{ml}$ streptomycin, and $10 \%$ FBS (HyClone, GE Healthcare) in a humidified incubator at $37^{\circ} \mathrm{C}$. Cells were made quiescent in DMEM/F-12 medium containing $0.5 \%$ serum and $0.01 \times$ amino acids (those found in the Gibco DMEM/F-12 were defined as $1 \times$ amino acids) for 6 hours before treatment with $1 \times$ amino acids, $100 \mathrm{nM}$ insulin, or vehicle (saline) alone for 30 minutes. The cells were quickly washed twice with ice-cold $\mathrm{Ca}^{2+} /$ $\mathrm{Mg}^{2+}$-free PBS and lysed on ice for 30 minutes in a lysis buffer containing 1\% Nonidet P-40, $50 \mathrm{mM} \mathrm{NaCl}, 20 \mathrm{mM}$ Tris-HCl, $137 \mathrm{mM} \mathrm{NaCl}$,
Figure 14. Schematic diagram depicting the regulation of kidney size by PI3K signaling in different contexts. UNX causes increased RBF and therefore increased renal delivery of free amino acids to the remaining kidney, leading to activation of a PTEN-independent, but class III PI3Kdependent, mTORC1 translocation to the lysosomal membrane where the mTORC1 activator RHEB resides and activates mTORC1 signaling to phosphorylate and activate the downstream effector S6K1. This leads to increased protein synthesis and renal hypertrophy, while the interplay between PTEN and EGFR-dependent class I PI3K/mTORC2/AKT/TSC2/ mTORC1/S6K1 signaling mediates appropriate kidney weight/BW ratios.

$1 \mathrm{mM} \mathrm{MgCl}, 1 \mathrm{mM} \mathrm{CaCl}, 10 \%$ glycerol, $1 \mathrm{mM} \mathrm{Na}_{3} \mathrm{VO}_{4}, 50 \mathrm{mM} \mathrm{NaF}$, $1 \mathrm{mM}$ PMSF, $10 \mu \mathrm{g} / \mathrm{ml}$ aprotinin, and $10 \mu \mathrm{g} / \mathrm{ml}$ leupeptin. Cell lysates were clarified at 19,400 $\mathrm{g}$ for 5 minutes at $4^{\circ} \mathrm{C}$, and protein concentrations were determined by the bicinchoninic acid assay (Pierce, Life Technologies). The cell lysates were subjected to immunoblot analysis and a class III PI3K kinase activity assay.

RNA interference. The siRNA transfection procedures for the mouse class III PI3K (encoded by the gene Pik3c3) RNA interference were performed as we described previously (66). Briefly, $70 \%$ to $80 \%$ confluent MCT cells were transfected with Silencer Negative Control siRNAs (catalog AM4611; Ambion, Life Technologies), Dharmacon SMARTpool ON-TARGETplus Mouse Pik3c3 siRNA L-063416-000005 (Thermo Fisher Scientific), or individual class III PI3K-specific siRNAs using the Lipofectamine reagent method (Invitrogen, Life Technologies). The 2 sets of individual siRNAs specifically target the following coding sequences of the mouse class III PI3K gene Pik3c3: GTGGCTGAAACTTCCGGTGAA and ATGGTGACGAATCATCTCCAA (siRNAs targeting these corresponding sequences of the human class III PI3K gene PIK3C3 have also been demonstrated to silence human class III PI3K effectively; ref. 43). Forty-eight hours after transfection, cells were made quiescent and treated as described above in Cell culture and treatment.

Class III PI3K kinase activity assay. A class III PI3K kinase activity assay was performed as previously described $(43,44)$, class III PI3K was immunoprecipitated from either MCT cell lysates or kidney homogenates, washed twice sequentially with $1 \% \mathrm{NP}-40 / 100 \mathrm{mM}$ $\mathrm{NaCl} / 1 \mathrm{mM} \mathrm{Na}_{3} \mathrm{VO}_{4} ; 500 \mathrm{mM} \mathrm{LiCl} / 100 \mathrm{mM}$ Tris-HCl (pH 7.4) $/ 1 \mathrm{mM}$ $\mathrm{Na}_{3} \mathrm{VO}_{4} ; 20$ mM Tris- $\mathrm{HCl}$ ( $\mathrm{pH}$ 7.4)/100 mM NaCl/1 mM EDTA/1 mM $\mathrm{Na}_{3} \mathrm{VO}_{4}$; and $20 \mathrm{mM}$ Tris- $\mathrm{HCl}$ (pH 7.4)/100 mM NaCl/10 mM MnCl before in vitro lipid kinase reactions using $\mathrm{PI}$ as a substrate in the presence of $\mathrm{MnCl}_{2}$ and $\left[\gamma^{-32} \mathrm{P}\right]$ ATP. The reactions were stopped by addition of $1 \mathrm{M} \mathrm{HCl}$, and then lipids were extracted by addition of an equal volume of $\mathrm{CHCl}_{3} / \mathrm{MeOH}$ (1:1) and analyzed by TLC with a mobile phase consisting of $\mathrm{CHCl}_{3} / \mathrm{MeOH} / 4 \mathrm{M} \mathrm{NH}_{4} \mathrm{OH}$ (9:7:2, v/v). The TLC plates were dried by incubation at $100^{\circ} \mathrm{C}$ for 5 minutes. The radioactive phosphorylation product PI-3-P was then visualized by autoradiography.

Statistics. Data are presented as the mean \pm SEM for at least 3 separate experiments (each performed in duplicate or triplicate). A 1-tailed unpaired $t$ test was used for statistical analysis, and ANOVA with Bonferroni's $t$ correction was used for multiple group comparisons. Mann-Whitney $U$ tests were used for statistical analysis of the quantitative data on the proximal tubular area, glomerular area, and ratios of Ki67-positive tubular nuclei to total number of tubular cells. A $P$ value of less than 0.05 was considered statistically significant.

Study approval. Animal care and all experimental procedures involving mice were performed in accordance with NIH guidelines for 
the care and use of live animals and were approved by the IACUCs of Vanderbilt University and Georgia Regents University.

\section{Acknowledgments}

This work was supported by the American Heart Association Scientist Development Grant 0630274N, NIH R01 grant DK83575, and Start-up Funds from Georgia Regents University Augusta (to J.-K. Chen); a VA Merit Award and NIH grants DK38226 and DK51265 (to R.C. Harris); and NIH grant DK46282 (to E.G. Neilson). K. Nagai was supported by funds from Mochida Memorial Foundation for Medical and Pharmaceutical Research and by Uehara Memorial Foundation and Grant-in-Aid for Research
Activity Start-up from the Ministry of Education, Culture, Sports, Science and Technology of Japan.

Address correspondence to: Jian-Kang Chen, Departments of Cellular Biology \& Anatomy and Medicine, Medical College of Georgia, Georgia Regents University, 1459 Laney Walker Boulevard, CB 2200, Augusta, Georgia 30912, USA. Phone: 706.721.8424; E-mail: jchen@gru.edu. Or to: Raymond C. Harris, C3121 MCN, Division of Nephrology, Department of Medicine, Vanderbilt University School of Medicine and Nashville Veterans Affairs Hospital, Nashville, Tennessee 37232, USA. Phone: 615.322.2150; E-mail: ray.harris@vanderbilt.edu.
1. Tomashefsky P, Tannenbaum M. Macromolecular metabolism in renal compensatory hypertrophy. I. Protein synthesis. Lab Invest. 1969;21(4):358-364.

2. Harris RC, Neilson EG. Toward a unified theory of renal progression. Annu Rev Med. 2006;57:365-380.

3. Silber S, Malvin RL. Compensatory and obligatory renal growth in rats. Am J Physiol. 1974;226(1):114-117.

4. Goss RJ. Effects of maternal nephrectomy on foetal kidneys. Nature. 1963;198:1108-1109.

5. Li J, et al. PTEN, a putative protein tyrosine phosphatase gene mutated in human brain, breast, and prostate cancer. Science. 1997;275(5308):1943-1947.

6. Myers MP, et al. P-TEN, the tumor suppressor from human chromosome 10q23, is a dual-specificity phosphatase. Proc Natl Acad Sci U S A. 1997;94(17):9052-9057.

7. Tamura M, Gu J, Matsumoto K, Aota S, Parsons R, Yamada KM. Inhibition of cell migration, spreading, and focal adhesions by tumor suppressor PTEN. Science. 1998;280(5369):1614-1617.

8. Myers MP, et al. The lipid phosphatase activity of PTEN is critical for its tumor supressor function. Proc Natl Acad Sci U S A. 1998;95(23):13513-13518.

9. Sun $\mathrm{H}$, et al. PTEN modulates cell cycle progression and cell survival by regulating phosphatidylinositol 3,4,5,-trisphosphate and Akt/protein kinase B signaling pathway. Proc Natl Acad Sci US A. 1999;96(11):6199-6204.

10. Stambolic V, et al. Negative regulation of PKB/ Akt-dependent cell survival by the tumor suppressor PTEN. Cell. 1998;95(1):29-39.

11. Alessi DR, et al. Characterization of a 3-phosphoinositide-dependent protein kinase which phosphorylates and activates protein kinase $\mathrm{B} \alpha$. Curr Biol. 1997;7(4):261-269.

12. Stephens L, et al. Protein kinase B kinases that mediate phosphatidylinositol 3,4,5-trisphosphate-dependent activation of protein kinase B. Science. 1998;279(5351):710-714.

13. Sarbassov DD, Guertin DA, Ali SM, Sabatini DM. Phosphorylation and regulation of Akt/ PKB by the rictor-mTOR complex. Science. 2005;307(5712):1098-1101.

14. Jean S, Kiger AA. Classes of phosphoinositide 3-kinases at a glance. J Cell Sci. 2014; 127(pt 5):923-928.

15. Yoon MS, Du G, Backer JM, Frohman MA, Chen J. Class III PI-3-kinase activates phospholipase D in an amino acid-sensing mTORC1 pathway. JCell Biol. 2011;195(3):435-447.

16. Laplante M, Sabatini DM. mTOR signaling in growth control and disease. Cell. 2012; 149(2):274-293

17. Sarbassov DD, et al. Rictor, a novel binding partner of $\mathrm{mTOR}$, defines a rapamycin-insensitive and raptor-independent pathway that regulates the cytoskeleton. Curr Biol. 2004;14(14):1296-1302.

18. Lawlor MA, Alessi DR. PKB/Akt: a key mediator of cell proliferation, survival and insulin responses? J Cell Sci. 2001;114(pt 16):2903-2910.

19. Kwiatkowski DJ. Tuberous sclerosis: from tubers to mTOR. Ann Hum Genet. 2003;67(pt 1):87-96.

20. Menon S, et al. Spatial control of the TSC complex integrates insulin and nutrient regulation of mTORC1 at the lysosome. Cell. 2014;156(4):771-785.

21. Potter CJ, Pedraza LG, Xu T. Akt regulates growth by directly phosphorylating Tsc2. Nat Cell Biol. 2002;4(9):658-665.

22. Chen JK, Chen J, Neilson EG, Harris RC. Role of mammalian target of rapamycin signaling in compensatory renal hypertrophy. JAm Soc Nephrol. 2005;16(5):1384-1391.

23. Chen JK, Chen J, Thomas G, Kozma SC, Harris RC. S6 Kinase 1 knockout inhibits uninephrectomy- or diabetes-induced renal hypertrophy. Am JPhysiol Renal Physiol. 2009;297(3):F585-F593.

24. Xu J, Chen J, Dong Z, Meyuhas O, Chen JK. Phosphorylation of ribosomal protein $\mathrm{S} 6$ mediates compensatory renal hypertrophy. Kidney Int . 2015;87(3):543-556.

25. Kim D, Dressler GR. PTEN modulates GDNF/ RET mediated chemotaxis and branching morphogenesis in the developing kidney. Dev Biol. 2007;307(2):290-299.

26. Di Cristofano A, Pesce B, Cordon-Cardo C, Pandolfi PP. Pten is essential for embryonic development and tumour suppression. Nat Genet. 1998;19(4):348-355.

27. Lesche R, et al. Cre/loxP-mediated inactivation of the murine Pten tumor suppressor gene. Genesis. 2002;32(2):148-149.

28. Iwano M, Plieth D, Danoff TM, Xue C, Okada $\mathrm{H}$, Neilson EG. Evidence that fibroblasts derive from epithelium during tissue fibrosis. J Clin Invest. 2002;110(3):341-350.

29. Jacquemin E, et al. Pattern of expression of gamma-glutamyl transpeptidase in rat liver and kidney during development: study by immunochemistry and in situ hybridization. J Pediatr
Gastroenterol Nutr. 1990;11(1):89-95.

30. Gan X, Wang J, Su B, Wu D. Evidence for direct activation of mTORC2 kinase activity by phosphatidylinositol 3,4,5-trisphosphate. J Biol Chem. 2011;286(13):10998-11002.

31. Johnson HA, Vera Roman JM. Compensatory renal enlargement. Hypertrophy versus hyperplasia. Am J Pathol. 1966;49(1):1-13.

32. Horie Y, et al. Hepatocyte-specific Pten deficiency results in steatohepatitis and hepatocellular carcinomas. JClin Invest. 2004;113(12):1774-1783.

33. Shioi $\mathrm{T}$, et al. Akt/protein kinase B promotes organ growth in transgenic mice. Mol Cell Biol. 2002;22(8):2799-2809.

34. Al-Awqati Q, Preisig PA. Size does matter: will knockout of p21(WAF1/CIP1) save the kidney by limiting compensatory renal growth? Proc Natl Acad Sci U S A. 1999;96(19):10551-10553.

35. Wolf G. Molecular mechanisms of renal hypertrophy: role of p27Kip1. Kidney Int. 1999;56(4):1262-1265.

36. Brown EJ, et al. A mammalian protein targeted by G1-arresting rapamycin-receptor complex. Nature. 1994;369(6483):756-758.

37. Jacinto E, et al. Mammalian TOR complex 2 controls the actin cytoskeleton and is rapamycin insensitive. Nat Cell Biol. 2004;6(11):1122-1128.

38. Zeng F, Singh AB, Harris RC. The role of the EGF family of ligands and receptors in renal development, physiology and pathophysiology. Exp Cell Res. 2009;315(4):602-610.

39. Chen J, Chen JK, Neilson EG, Harris RC. Role of EGF receptor activation in angiotensin II-induced renal epithelial cell hypertrophy. JAm Soc Nephrol. 2006;17(6):1615-1623.

40. Chen J, et al. EGFR signaling promotes TGFbeta-dependent renal fibrosis. J Am Soc Nephrol. 2012;23(2):215-224.

41. Pearson RB, et al. The principal target of rapamycin-induced p70s6 $\mathrm{k}$ inactivation is a novel phosphorylation site within a conserved hydrophobic domain. ЕMBO J. 1995;14(21):5279-5287.

42. Burnett PE, Barrow RK, Cohen NA, Snyder SH, Sabatini DM. RAFT1 phosphorylation of the translational regulators p70 S6 kinase and 4E-BP1. Proc Natl Acad Sci U S A. 1998;95(4):1432-1437.

43. Nobukuni T, et al. Amino acids mediate mTOR/ raptor signaling through activation of class 3 phosphatidylinositol 3OH-kinase. Proc Natl Acad Sci U S A. 2005;102(40):14238-14243.

44. Byfield MP, Murray JT, Backer JM. hVps34 
is a nutrient-regulated lipid kinase required for activation of p70 56 kinase. J Biol Chem. 2005;280(38):33076-33082.

45. Sancak Y, et al. The Rag GTPases bind raptor and mediate amino acid signaling to mTORC1. Science. 2008;320(5882):1496-1501.

46. Sancak Y, Bar-Peled L, Zoncu R, Markhard AL, Nada S, Sabatini DM. Ragulator-Rag complex targets mTORC1 to the lysosomal surface and is necessary for its activation by amino acids. Cell. 2010;141(2):290-303.

47. Eskelinen EL. Roles of LAMP-1 and LAMP-2 in lysosome biogenesis and autophagy. Mol Aspects Med. 2006;27(5):495-502.

48. Buse MG, Reid SS. Leucine. A possible regulator of protein turnover in muscle. JClin Invest. 1975;56(5):1250-1261.

49. Li JB, Jefferson LS. Influence of amino acid availability on protein turnover in perfused skeletal muscle. Biochim Biophys Acta. 1978;544(2):351-359.

50. Wang X, Campbell LE, Miller CM, Proud CG. Amino acid availability regulates p70 66 kinase and multiple translation factors. Biochem J. 1998;334(pt 1):261-267.

51. Hara K, Yonezawa K, Weng QP, Kozlowski MT, Belham C, Avruch J. Amino acid sufficiency and mTOR regulate p70 56 kinase and eIF-4E BP1 through a common effector mechanism. J Biol Chem. 1998;273(23):14484-14494.
52. Sigmon DH, Gonzalez-Feldman E, Cavasin MA, Potter DL, Beierwaltes WH. Role of nitric oxide in the renal hemodynamic response to unilateral nephrectomy. JAm Soc Nephrol. 2004;15(6):1413-1420.

53. Harris RC, Seifter JL, Brenner BM. Adaptation of $\mathrm{Na}^{+}-\mathrm{H}^{+}$exchange in renal microvillus membrane vesicles. Role of dietary protein and uninephrectomy. J Clin Invest. 1984;74(6):1979-1987.

54. Chen J, Chen MX, Fogo AB, Harris RC, Chen JK. $\mathrm{mVps} 34$ deletion in podocytes causes glomerulosclerosis by disrupting intracellular vesicle trafficking. J Am Soc Nephrol. 2013;24(2):198-207.

55. Rankin EB, Tomaszewski JE, Haase VH. Renal cyst development in mice with conditional inactivation of the von Hippel-Lindau tumor suppressor. Cancer Res. 2006;66(5):2576-2583.

56. Nowinski WW. Early history of renal hypertrophy. In: Nowinski WW, Goss RJ, eds. Compesatory Renal Hypertrophy. New York, New York, USA: Academic Press; 1969:1-8.

57. Preisig PA. Renal hypertrophy and hyperplasia. In: Selden DW, Giebisch G, eds. The Kidney. Philadelphia, Pennsylvania, USA: Lippincott Williams \& Wilkins; 2000:727-748.

58. Brenner BM. Remission of renal disease: recounting the challenge, acquiring the goal. J Clin Invest. 2002;110(12):1753-1758.

59. Hostetter TH. Progression of renal disease and renal hypertrophy. Annu Rev Physiol. 1995;57:263-278.

60. Brenner BM, Mackenzie HS. Nephron mass as a risk factor for progression of renal disease. Kidney Int Suppl. 1997;63:S124-S127.

61. Schu PV, Takegawa K, Fry MJ, Stack JH, Waterfield MD, Emr SD. Phosphatidylinositol 3-kinase encoded by yeast VPS34 gene essential for protein sorting. Science. 1993;260(5104):88-91.

62. Chen JK, Falck JR, Reddy KM, Capdevila J, Harris RC. Epoxyeicosatrienoic acids and their sulfonimide derivatives stimulate tyrosine phosphorylation and induce mitogenesis in renal epithelial cells. J Biol Chem. 1998;273(44):29254-29261.

63 . Bhowmick NA, et al. TGF- $\beta$ signaling in fibroblasts modulates the oncogenic potential of adjacent epithelia. Science. 2004;303(5659):848-851.

64. Deger SM, et al. Insulin resistance and protein metabolism in chronic hemodialysis patients. J Ren Nutr. 2013;23(3):e59-e66.

65. Haverty TP, et al. Characterization of a renal tubular epithelial cell line which secretes the autologous target antigen of autoimmune experimental interstitial nephritis. J Cell Biol. 1988;107(4):1359-1368.

66. Chen J, et al. Mitogenic activity and signaling mechanism of 2-(14,15- epoxyeicosatrienoyl) glycerol, a novel cytochrome $\mathrm{p} 450$ arachidonate metabolite. Mol Cell Biol. 2007;27(8):3023-3034. 